\title{
QPCT regulation by CTCF leads to sunitinib resistance in renal cell carcinoma by promoting angiogenesis
}

\author{
TANGLIANG ZHAO ${ }^{1 *}$, YULIN ZHOU $^{1,2^{*}}$, QINGYUN WANG $^{1 *}$, XIAOMING YI $^{1}$, SILUN GE $^{1}$, HAOWEI HE $^{1}$, \\ SONG XUE ${ }^{1}$, BOWEN DU $^{1}$, JINGPING GE ${ }^{1}$, JIE DONG ${ }^{1}$, LE QU $^{1}$, LINHUI WANG $^{3}$ and WENQUAN ZHOU ${ }^{1}$ \\ ${ }^{1}$ Department of Urology, Jinling Hospital, Medical School of Nanjing University, Nanjing, Jiangsu 210002; \\ ${ }^{2}$ Department of Urology, Xuzhou Central Hospital, Medical College of Soochow University, Suzhou, Jiangsu 215123; \\ ${ }^{3}$ Department of Urology, Changzheng Hospital, Second Military Medical University, Shanghai 200003, P.R. China
}

Received January 18, 2021; Accepted May 6, 2021

DOI: $10.3892 /$ ijo.2021.5228

\begin{abstract}
Sunitinib is widely used as a first-line treatment for advanced renal cell carcinoma (RCC). However, a number of patients with RCC who receive sunitinib develop drug resistance; and the biological mechanisms involved in resistance to sunitinib remain unclear. It has previously been suggested that the protein glutaminyl-peptide cyclotransferase (QPCT) is closely related to sunitinib resistance in RCC. Thus, in the present study, in order to further examine the molecular mechanisms responsible for sunitinib resistance in RCC, sunitinib-non-responsive and -responsive RCC tissue and plasma samples were collected and additional experiments were performed in order to elucidate the molecular mechanisms responsible for sunitinib resistance in RCC. The upstream and downstream regulatory mechanisms of QPCT were also evaluated. On the whole, the data from the present study suggest that QPCT, CCCTC-binding factor (CTCF)
\end{abstract}

Correspondence to: Professor Wenquan Zhou, Department of Urology, Jinling Hospital, Medical School of Nanjing University, 305 East Zhongshan Road, Nanjing, Jiangsu 210002, P.R. China E-mail: shzwqzsl@163.com

Professor Linhui Wang, Department of Urology, Changzheng Hospital, Second Military Medical University, 415 Fengyang Road, Shanghai 200003, P.R. China

E-mail: wanglinhui@smmu.edu.cn

*Contributed equally

Abbreviations: RCC, renal cell carcinoma; QPCT, glutaminyl peptide cyclotransferase; PIK3CA, phosphatidylinositol4,5-bisphosphate 3-kinase catalytic subunit alpha; HUVECs, human umbilical vein endothelial cells; VEGF, vascular endothelial growth factor; PFS, progression-free survival; PCR, polymerase chain reaction; IHC, immunohistochemistry; ChIP, chromatin immunoprecipitation; Co-IP, co-immunoprecipitation

Key words: glutaminyl-peptide cyclotransferase, CCCTC-binding factor, phosphatidylinositol-4,5-bisphosphate 3-kinase catalytic subunit alpha, sunitinib and phosphatidylinositol-4,5-bisphosphate 3-kinase catalytic subunit alpha (PIK3CA) may be used as targets for predicting, reversing and treating sunitinib-resistant RCC.

\section{Introduction}

Renal cell carcinoma ( $\mathrm{RCC}$ ) is one of the most common malignant tumors of the urinary system, accounting for 2-3\% of adult malignancies (1). The incidence of $\mathrm{RC}$ is increasing each year (2). The early diagnosis of RCC is challenging; 20-30\% of patients with RCC are diagnosed with metastases at the time of initial diagnosis (3-5). Surgical resection remains the most effective treatment for early-stage RCC, while immunotherapy and radiotherapy are commonly used for advanced-stage carcinoma. However, these treatment methods are associated with severe side-effects $(6,7)$. Recently, targeted therapy has become increasingly popular for treating patients with RCC.

The receptor tyrosine kinase inhibitors (TKIs) represented by sunitinib are the first-line treatment approach for advanced-stage RCC (4). Sunitinib is known to exhibit antitumor and anti-angiogenic activities. It blocks vascular endothelial growth factor receptor (VEGFR), platelet-derived growth factor receptor (PDGFR), KIT and other important molecules affecting cell growth and survival (8-11). Although targeted drugs have greatly improved the therapeutic prospects of advanced-stage RCC, drug resistance has gradually reduced the clinical effects of targeted drugs. Approximately $20 \%$ of patients with RCC prescribed with sunitinib have been reported to develop innate resistance, while the majority of patients develop secondary resistance after 6-11 months $(12,13)$. The molecular and biological mechanisms involved in sunitinib resistance remain unclear, and there are no effective biomarkers for predicting resistance, at least to the best of our knowledge.

In a previous study, the authors screened out the protein glutaminyl-peptide cyclotransferase (QPCT), which is closely related to sunitinib resistance in RCC, using high-throughput sequencing data and subsequent validation experiments (14). The QPCT gene encodes glutamyl peptidyltransferase, which modifies the protein by transforming $\mathrm{N}$-terminal glutamic acid into pyroglutamine. This renders proteins more resistant to protease degradation, becoming more hydrophobic and neurotoxic, and thus easier to aggregate (15). In the present study, in 
order to further elucidate the molecular mechanisms responsible for sunitinib resistance in RCC, sunitinib-non-responsive and -responsive RCC tissue and plasma samples were collected, and additional experiments were performed to elucidate the molecular mechanisms responsible for sunitinib resistance in RCC.

\section{Materials and methods}

Patients with RCC and clinical samples. Patients with RCC who underwent surgical resection prior to receiving adjuvant therapy at Jinling Hospital (Nanjing, China) from 2010 to 2018 were enrolled in the present study. All patients signed informed consent forms before participating in the study, and the study was approved by the Ethics Committee of Jinling Hospital. The accession number for this approval was 2020DZGZRZX-008. The expression of QPCT was detected in 20 pairs of sunitinib-resistant and -sensitive RCC tissues. The details of the patients are presented in Table SI.

To investigate the association between QPCT expression and sunitinib responsivity, tissue samples were collected from 128 patients (including the 20 pairs of sunitinib-resistant and -sensitive RCC tissues mentioned in Table SI) with advanced clear cell RCC (ccRCC) who received no other treatment between July, 2010 and February, 2018. The sunitinib group $(n=72)$ received at least two cycles of targeted therapy, while the control group $(n=56)$ received no treatment. These ccRCC tissues were constructed into a tissue microarray, and the expression of QPCT was detected by immunohistochemistry. The details of the patients are presented in Table SII.

To investigate the association between the QPCT content in peripheral blood of patients with RCC and sunitinib reactivity, plasma samples from patients (including the 20 pairs of patient plasma samples mentioned in Table SI) with sunitinib resistance and sensitivity were collected at Jinling Hospital from 2010 to 2018. The details of the patients are presented in Table SIII.

Cell lines and reagents. Human RCC cell lines, including OS-RC-2 (cat. no. TCHu40), A498 (cat. no. HTB-44), 786-O (cat. no. TCHu186), ACHN (cat. no. TCHu199), KETR-3 (cat. no. CRL-1161) and human umbilical vein endothelial cells (HUVECs, cat. no. CRL-1730) were obtained from the Chinese Academy of Sciences (Shanghai, China) or the American Type Culture Collection (ATCC). The A498 and ACHN cells were cultured in MEM (10-010-CV, Corning, Inc.) supplemented with $10 \%$ fetal bovine serum (FBS, 16000044, Gibco; Thermo Fisher Scientific, Inc.), and the other RCC cells were cultured in RPMI-1640 (10-040-CV, Corning, Inc.) supplemented with 10\% FBS. HUVECs were cultured in DMEM (Corning, Inc.) containing 10\% FBS. The cells were grown in a single layer on a plastic cell culture dish in humidified air containing $5 \% \mathrm{CO}_{2}$ at $37^{\circ} \mathrm{C}$. Sunitinib was purchased from Shanghai Selleck Chemicals Co., Ltd. MG132 and cycloheximide (CHX) were obtained from Apexbio Technology, LLC. Recombinant human glutamine peptide loop transferase/QPCT (6368-Zn) was obtained from R\&D Systems. Matrigel matrix basement membrane matrix (354234, BD Biosciences) was purchased from Corning, Inc.
Animal experiments. A total of $16 \mathrm{BALB} / \mathrm{c}$ male nude mice, 4 weeks old, weighing $20 \mathrm{~g}$, were obtained from the Shanghai Institute of Material Medical (Chinese Academy of Science, Shanghai, China). The mice were maintained under pathogen-free conditions in accordance with relevant guidelines and regulations for the care and use of laboratory animals, with the approval of the Institutional Animal Care and Use Committee at Jinling Hospital and the accession number for this approval was 2020JLHGKJDWLS-47.

A total of $7 \times 10^{6} \mathrm{lv}$-PIK3CA and $1 \mathrm{v}-\mathrm{NC} 786-\mathrm{O}$ cells (obtained via transfection as described below) were subcutaneously inoculated into the left and right side of male athymic $\mathrm{BALB} / \mathrm{c}$ nude mice (4 weeks old). All the mice were housed in an environment with a temperature of $22 \pm 1^{\circ} \mathrm{C}$, a relative humidity of $50 \pm 1 \%$ and a light/dark cycle of $12 / 12 \mathrm{~h}$. The mice had free access to food and water. At one week after the injection of tumor cells, the animals were randomly assigned to the control or experimental groups ( $\mathrm{n}=4 \mathrm{mice} / \mathrm{group})$. When the xenografts reached $100 \mathrm{~mm}^{3}$, sunitinib (40 mg/kg/day) or saline (control) was used for intragastric administration. Tumor size was monitored at five-day intervals. Xenograft tumor volumes were measured using a vernier caliper and individually calculated using the following formula: Volume $=\mathrm{axb}^{2} / 2$ ('a' represents length and 'b' represents width). Xenograft tumor samples were collected for histological evaluation (paraffin-embedded sections) or were snap-frozen in liquid nitrogen. A total of 16 mice participated in the experiment and no mice died during the experiment. The animal experiment lasted for eight weeks and the mice were sacrificed eight weeks following inoculation. The mice were anesthetized by an inhalation of isoflurane. Isoflurane was added into the evaporator of an anesthesia machine and the percentage of isoflurane was adjusted in the mixed gas (the concentration of isoflurane was $5 \%$ ). After $\sim 1 \mathrm{~min}$, the mice were placed in the induction box. The induction box was then closed and the mice were fully anesthetized, which took $\sim 2 \mathrm{~min}$. The induction box was gently shaken to determine whether the mice were completely anesthetized. If the bodies of mice turned over to the side position and the mice did not try to resume the prone position, this indicated that the mice were completely anesthetized. The mice were then sacrificed by cervical dislocation under anesthesia to reduce their pain. All animal experiments (including the mouse euthanasia procedure) were conducted according to the AAALAC and the IACUC guidelines.

RNA extraction, $c D N A$ preparation and reverse transcriptionquantitative polymerase chain reaction ( $R T-q P C R)$. Total RNA was extracted from cells and tissues using TRIzol reagent (Takara Bio, Inc.), according to the manufacturer's instructions. Total RNA quality was assessed using a Nanodrop 2000 and agarose gel electrophoresis. First-strand cDNA was generated from $2 \mu \mathrm{g}$ of total RNA using M-MLV reverse transcriptase (Invitrogen; Thermo Fisher Scientific, Inc.) with random primers. Quantitative PCR (qPCR) was performed on triplicate samples in a reaction mix of SYBR-Green (Takara Bio, Inc.) using the ABI 7900HT Fast Real-Time PCR System (Applied Biosystems; Thermo Fisher Scientific, Inc.). The conditions of PCR denaturation, annealing and extension were respectively $94^{\circ} \mathrm{C} 60 \mathrm{sec}, 37^{\circ} \mathrm{C} 60 \mathrm{sec}$, and $72^{\circ} \mathrm{C}$ $120 \mathrm{sec}$. The expression of indicated genes was normalized 
to the endogenous reference control, $\beta$-actin, using the $2^{-\Delta \Delta \mathrm{Cq}}$ method (16). The primers were synthesized by Sangon Biotech (Shanghai) Co., Ltd. Each qRT-PCR reaction was performed in triplicate. The primer sequences were as follows: QPCT forward, 5'-AAATTGCAGAAGGCACCAGT-3' and reverse, 5'-CTGAATTCGCTGCATGATGT-3'; CCCTC-binding factor (CTCF) forward, 5'-CTGCTGTGGACGATACCC-3' and reverse, 5'-GCAAGGCCCTCTTTAGAC-3'; phosphatidylinositol-4,5-bisphosphate 3-kinase catalytic subunit alpha (PIK3CA) forward, 5'-CATGCATTGTTTTGCACCCC-3' and reverse, 5'-ATGGAAGACGGGAGATTCACAT-3' and $\beta$-actin forward, 5'-CTGGTGCCTGGGGCG-3' and reverse, 5'-AGCCTCGCCTTTGCCGA-3'.

Western blot analysis. Briefly, RCC cells or tissues were lysed to obtain proteins using RIPA buffer (EMD Millipore). The BCA method was used to determine the protein concentration, Total RCC cell and RCC tissue lysates were prepared in $1 \mathrm{X}$ sodium dodecyl sulphate (SDS) buffer. Identical quantities of protein $(20 \mu \mathrm{l})$ were separated by $10 \%$ SDS gel electrophoresis and transferred onto nitrocellulose filter membranes. The membranes were blocked with $5 \%$ non-fat milk for $2 \mathrm{~h}$ at room temperature and incubated with specific antibodies overnight at $4^{\circ} \mathrm{C}$. Following incubation with antibodies specific for QPCT (ab201172, Abcam; 1:1,000), PIK3CA (ab40776, Abcam; 1:1,000), ubiquitin (3936, Cell Signaling Technology, Inc.; 1:1,000), AKT (4691, Cell Signaling Technology, Inc.; 1:1,000), p-AKT (4060, Cell Signaling Technology, Inc.; 1:1,000) and GAPDH (sc-25778; Santa Cruz Biotechnology, Inc.; 1:2,000), the blots were incubated with IRDye 800-conjugated goat anti-rabbit IgG (2095, Li-Cor Biosciences Inc.; 1:1,000) for $1 \mathrm{~h}$ in the dark at room temperature, and bands were detected using an Odyssey infrared scanner (Li-Cor). Odyssey software (V1.01, Li-Cor Biosciences Inc.) was used for densitometry. GAPDH was used as the loading control. Each western blot analysis experiment was repeated three times.

ELISA. A $25 \mathrm{ng} /$ well of capture antibody goat-anti QPCT (PA5-112679, ThermoFisher) was coated over night at $4^{\circ} \mathrm{C}$. The wells were blocked for $2 \mathrm{~h}$ by the addition of $200 \mu \mathrm{l}$ blocking buffer [protein free (TBS) blocking buffer (37570, Thermo Fisher Scientific, Inc.)] and then washed three times using $300 \mu \mathrm{l}$ of wash buffer [protein free T20 (TBS) blocking buffer (37571, Thermo Fisher Scientific, Inc.)]. Standard peptides (PeproTech, Inc.) and samples (human plasma) were diluted using dilution buffer [protein-free T20 (TBS) blocking buffer)] and $100 \mu 1$ were applied onto the test plate. The incubation of test samples and standard peptides was performed for $2 \mathrm{~h}$ at room temperature and the plate was then washed three times using wash buffer. Thereafter, wells were washed three times with $300 \mu \mathrm{l}$ of wash buffer and the chromogen SureBlue (KPL, Inc.) was applied in a volume of $100 \mu \mathrm{l}$ to each well and incubated in the dark. After $30 \mathrm{~min}$, the reaction was abrogated using $50 \mu \mathrm{l}$ Stop Solution (1.2 N H2SO4) and absorption was determined at $450 \mathrm{~nm}$. The absorbance was recorded at $450 \mathrm{~nm}$ using a microplate reader (Varioskan Flash; Thermo Fisher Scientific, Inc.). The reference wavelength of $550 \mathrm{~nm}$ was subtracted from sample absorption at $450 \mathrm{~nm}$.
Cell transfection and lentivirus infection. Transfections were performed using a Lipofectamine RNAiMAX Transfection Reagent kit (Invitrogen; Thermo Fisher Scientific, Inc.) according to the manufacturer's instructions. Small interfering RNAs and negative control RNAs were introduced into ACHN and OS-RC-2 cells at 75 pmol per well in six-well plates and the cells were grown in humidified air containing $5 \% \mathrm{CO}_{2}$ at $37^{\circ} \mathrm{C}$. The cells were harvested at $48 \mathrm{~h}$ following transfection. CTCF siRNA was synthesized by GenePharma, Inc., with a sequence of si-CTCT1, 5'-GUGGUACCAUGA AGAUGCATT-3' (forward) and 5'-UGCAUCUUCAUGGUA CCACTT-3' (reverse); si-CTCT2, 5'-GGCAAGACAUGC UGAUAAUTT-3' (forward) and 5'-AUUAUCAGCAUGUCU UGCCTT-3' (reverse). A non-silencing siRNA oligonucleotide that does not recognize any known mammalian gene homolog (GenePharma, Shanghai, China) was used as a negative control.

QPCT-overexpressing, PIK3CA-overexpressing and control lentiviruses were produced by Obio Technology (Shanghai) Corp., Ltd. The CDS sequence containing QPCT or PIK3CA was amplified by PCR and cloned into the lentiviral vectors, pLVX-CMV-QPCT-3FLAG-PGK-Puro or pLVX-CMV-PIK3CA-3FLAG-PGK-Puro, to construct the QPCT-overexpressing or PIK3CA-overexpressing lentiviruses. The concentration and purification of lentivirus wase divided into primary purification and ultracentrifugation. The concentrations of QPCT-overexpressing lentiviruses and PIK3CA-overexpressing lentiviruses were $7.17 \times 10^{8}$ and $5.26 \times 10^{8}$, respectively. The QPCT or PIK3CA knockdown lentiviruses and control lentiviruses were constructed for a siRNA. The lentiviral vector used was LKD001 pLKD-CMV-Puro-U6-shRNA. The concentration of QPCT knockdown lentiviruses and PIK3CA knockdown lentiviruses were $1.39 \times 10^{9}$ and $2.28 \times 10^{9}$, respectively.CTCF-overexpressing and control lentiviruses were produced by Hanbio Biotechnology Co., Ltd. The CDS sequence containing CTCF was amplified by PCR and cloned into the lentiviral vector pHBLV-CMV-MCS-3flag-EF1-puro to construct the $\mathrm{CTCF}$-overexpressing lentiviruses. The concentration of CTCF-overexpressing lentiviruses was $3.15 \times 10^{8}$. The appropriate amount of lentiviruses $(1: 1,000)$ was transfected into RCC cells. The cells were grown in humidified air containing $5 \% \mathrm{CO}_{2}$ at $37^{\circ} \mathrm{C}$ and the medium was changed after $48 \mathrm{~h}$. After the cells were infected with lentiviruses for $72 \mathrm{~h}, 1.5 \mu \mathrm{g} / \mathrm{ml}$ puromycin was selected for stable transformation screens. RT-qPCR was used to verify the transfection efficiency of the lentiviruses.

Immunohistochemistry. The sections were heated at $70^{\circ} \mathrm{C}$ for $1 \mathrm{~h}$, dewaxed in xylene, and dehydrated through a gradient concentration of alcohol. After retrieving and blocking endogenous peroxidase and non-specific staining with $3 \%$ $\mathrm{H}_{2} \mathrm{O}_{2}$ and normal bovine serum, the sections were incubated with primary antibody overnight at $4^{\circ} \mathrm{C}$. The slides were then incubated with horseradish peroxidase (HRP)-conjugated secondary antibody (HS101-01, TransGen Biotech, 1:500) for $10 \mathrm{~min}$ at $37^{\circ} \mathrm{C}$. Finally, the sections were visualized by diaminobenzidine (DAB) solution for $15 \mathrm{~min}$ at $37^{\circ} \mathrm{C}$ and then counterstained with hematoxylin. Two pathologists blinded to the patient outcomes independently scored the staining intensities and percentages of positive tumor cells. The results of 
immunohistochemistry were observed using an optical microscope (ZTX-3S-C2, AS ONE Corporation). Specimens were stained with antibodies to QPCT (ab201172, Abcam, 1:100), PIK3CA (ab135384, Abcam, 1:100), CD31 (ab28364, Abcam, 1:50) and CD34 (ab110643, Abcam, 1:100).

Chromatin immunoprecipitation. Chromatin immunoprecipitation was performed using the EZ ChIP Chromatin Immunoprecipitation kit for cell line samples (EMD Millipore) according to the manufacturer's instructions. 786-O and KETR-3 cells $\left(1 \times 10^{7}\right.$ cells) were cross-linked with $1 \%$ formaldehyde and incubated for $10 \mathrm{~min}$ at $37^{\circ} \mathrm{C}$. ChIP assay was performed according to the manufacturer's protocol using monoclonal Anti-CTCF antibody (ab128873, Abcam; 1:100) or normal rabbit IgG as a negative control (ab172730, Abcam, $1: 100)$. An aliquot of lysates $(20 \mu 1)$ was used as an input control. DNA enrichment was determined by quantitative PCR (qPCR), and was normalized to the input using the ABI 7900HT Fast Real-Time PCR System (Applied Biosystems; Thermo Fisher Scientific, Inc.). The sequence for Primerl (containing the CTCF binding QPCT site) was as follows: Forward, 5'-GTGTATTTCCAGGCAAGCCC-3' and reverse, 5'-CCACCCACTCACTCTGTCTTC-3'.

Human proteome microarray assay. The HuProt microarray assay $(17,18)$ and data analysis were performed by Wayen Biotechnologies (Shanghai), Inc., according to the following procedure. The HuProt microarray (CDI Laboratories, Inc.) comprises 20,240 human full-length proteins with N-terminal glutathione S-transferase (GST) tags. Human proteome microarrays (HuProtTM $20 \mathrm{~K}$ ) were blocked with blocking buffer (1\% BSA and $0.1 \%$ Tween-20 in TBST) for $1 \mathrm{~h}$ at room temperature with gentle agitation. The QPCT protein was labelled with biotin with an Antibody Array assay kit (Full Moon BioSystems, Inc.) and was then diluted to $0.01 \mathrm{mg} / \mathrm{ml}$ in blocking buffer and incubated on the blocked proteome microarray at room temperature for $1 \mathrm{~h}$. The microarrays were washed three times for 5 min each with TBST, incubated with streptavidin-Cy5 at a dilution of 1:1,000 (Thermo Fisher Scientific, Inc.) for $1 \mathrm{~h}$ at room temperature and subjected to three more 5-min washes. The microarrays were spun dry at 1,500 rpm for 3 min and subjected to scanning with a GenePix 4000B (Axon Instruments, Inc.) to visualize and record the results. GenePix Pro 6.0 was used for data analysis. GO (Biological Process, Molecular Function, Cellular Component) and KEGG_Pathway analysis were conducted for the proteins that bound to QPCT.

Co-immunoprecipitation. Co-immunoprecipitation (co-IP) was performed according to the manufacturer's instructions (Pierce Co-Immunoprecipitation kit, Thermo Fisher Scientific, Inc.). RCC cells with indicated treatment were used for one immunoprecipitation reaction. Briefly, cells were lysed in a series of buffers and centrifugation steps to obtain lysate supernatant. Indicated antibodies were covalently coupled onto an amine-reactive resin and used to bait the corresponding proteins. Antibodies against QPCT (sc-517122, Santa Cruz Biotechnology, Inc., 1:50) and PIK3CA (4255, Cell Signaling Technology, Inc.; 1:50) were incubated for $12 \mathrm{~h}$ at $4^{\circ} \mathrm{C}$ and then incubated with IRDye 800-conjugated goat anti-rabbit IgG
(2095, Li-Cor Biosciences Inc.; 1:1,000) for $1 \mathrm{~h}$ in the dark at room temperature, and bands were detected using an Odyssey infrared scanner (Li-Cor).

In vitro Matrigel tube formation assay. HUVECs $\left(5 \times 10^{5}\right.$ cells per well) were seeded onto Matrigel plates (containing $200 \mu \mathrm{l}$ Matrigel) and cultured for $12 \mathrm{~h}$ at $37^{\circ} \mathrm{C}$ in $5 \% \mathrm{CO}_{2}$. Capillary-like structures were evident and counted using a phase-contrast microscope (Shanghai Optical Instrument Factory), and the networks formed by HUVECs were quantified using ImageJ software V1.8.0.112 (National Institutes of Health). The group incubated with exogenous VEGF (RP-87723, Gibco; Thermo Fisher Scientific, Inc.; $10 \mu \mathrm{M}$ ) was used as the positive control group and the purified QPCT cytokines (rhQPCT, 6368-ZN-010, R\&D Systems, Inc.; $10 \mu \mathrm{M}$ ) was used.

Statistical analysis. SPSS 22.0 software (SPSS, Inc.) was used for all statistical analyses in the present study. Data are expressed as the mean \pm standard deviation (SD). Depending on the type of data, the appropriate statistical methods were used. A two-tailed t-test or non-parametric Mann-Whitney U test was used for comparisons between two groups. Analysis of variance or Kruskal-Wallis was used for comparisons among multiple groups. Dunnett's test was used for post hoc evaluation analysis. Pearson Chi-squared test was applied to analyze clinical variables. Kaplan-Meier survival analysis was used to compare the effects of QPCT dichotomous expression on the survival rate of patients with RCC using the log-rank test or Renyi test if the hazard rates crossed. A P-value $<0.05$ was considered to indicate a statistically significant difference.

\section{Results}

QPCT expression is increased in the sunitinib-non-responsive $R C C$ tissues and plasma, and patients with RCC with a high QPCT expression have a poor response to sunitinib. The present study first selected 20 pairs of sunitinib-non-responsive and -responsive RCC tissue samples to detect QPCT expression at the mRNA and protein level. It was found that QPCT expression was upregulated in the sunitinib-non-responsive RCC tissues (Fig. 1A and B). Immunohistochemistry was then performed to detect QPCT expression in a tissue microarray, including 128 RCC tissue samples. Once again, QPCT expression was upregulated in the sunitinib-nonresponsive RCC tissues (Fig. 1C).

QPCT can be secreted by tumor cells $(14,15)$. ELISA of patients with RCC who had a favorable or adverse response to sunitinib therapy revealed elevated plasma QPCT levels in patients who did not respond to sunitinib (Fig. 1D). Since QPCT was functionally involved in the sunitinib reaction of RCC cells, the expression of QPCT in the tissue microarray including 128 RCC samples was detected by immunohistochemistry, and the association between the QPCT content and $\mathrm{RCC}$ response to sunitinib treatment was analyzed by combining the prognostic information of the patients. It was found that sunitinib treatment extended the progression-free survival (PFS) of patients with RCC (Fig. 1E, left panel), while patients with a low QPCT expression in tumor tissue had a 
A

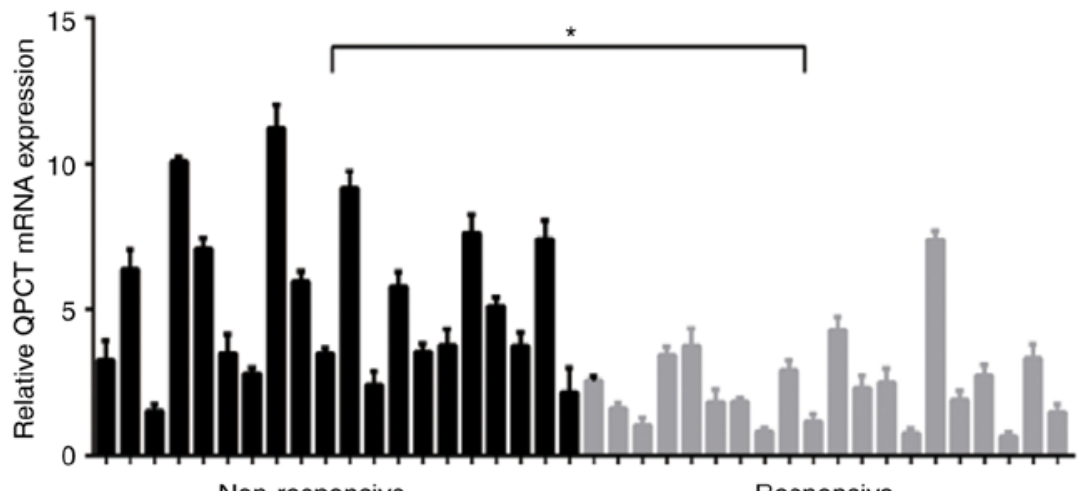

Non-responsive

Responsive
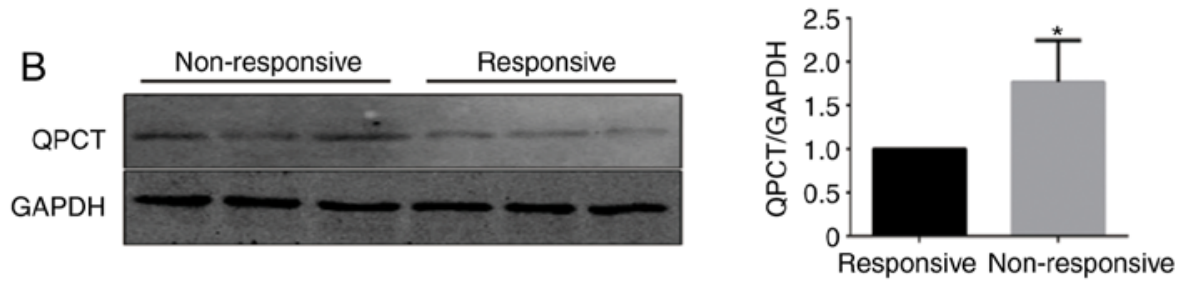

C

N1

N2

N3
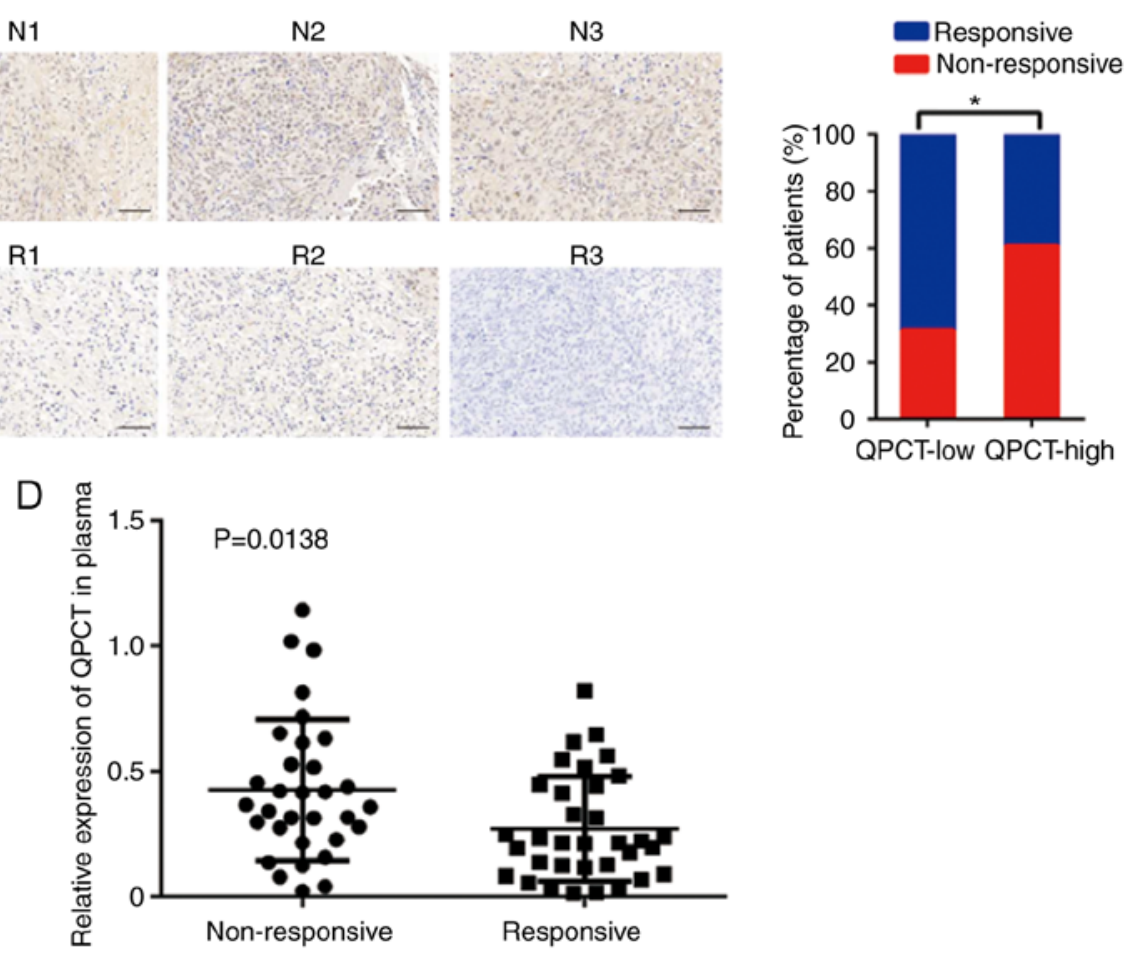

E

All patients
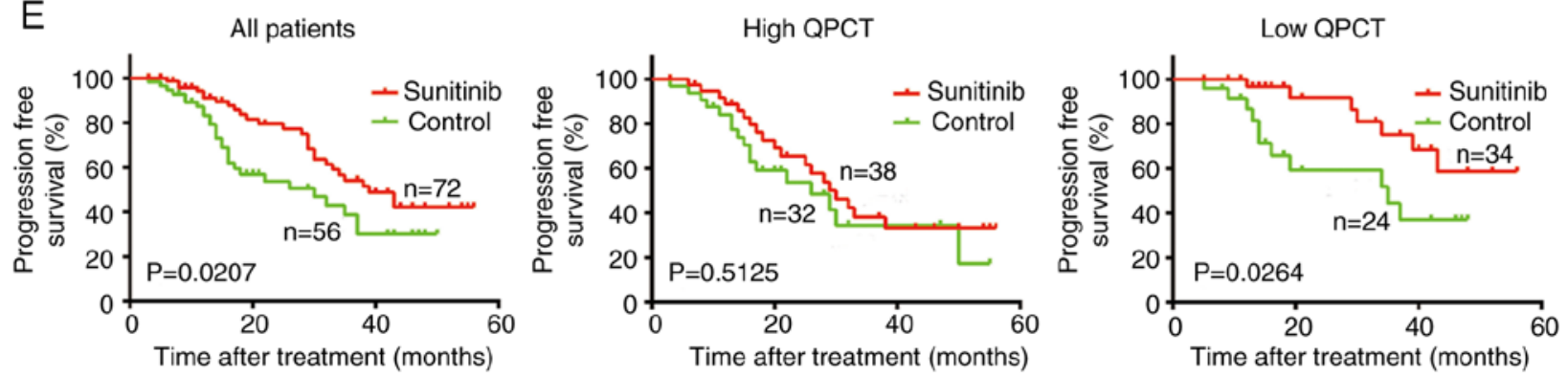

Figure 1. In the sunitinib-non-responsive RCC tissues and plasma, QPCT expression is increased, and patients with RCC with a high QPCT expression have a poor response to sunitinib. (A) mRNA expression of QPCT in 20 pairs of sunitinib-non-responsive and -responsive RCC tissues. (B) Results of western blot analysis of QPCT protein in 20 pairs of sunitinib nonresponsive and responsive RCC tissues. (C) Representative immunohistochemical results of QPCT expression in sunitinib-non-responsive and -responsive RCC tissues (scale bar, $100 \mu \mathrm{m}$; left panel), and percentage of samples nonresponsive and responsive to sunitinib at different QPCT levels (right panel). (D) ELISA of plasma QPCT levels in patients with RCC at Jinling Hospital. (E) Kaplan-Meier analysis of PFS for all patients $(\mathrm{P}=0.0207)$ (left panel). Kaplan-Meier analysis of PFS in patients with a high QPCT expression ( $\mathrm{P}=0.5125)$ (middle panel). Kaplan-Meier analysis of PFS in patients with low QPCT expression $(\mathrm{P}=0.0264)$ (right panel). The results are presented as the mean $\pm \mathrm{SD}$. "P $<0.05$. RCC, renal cell carcinoma; QPCT, glutaminyl peptide cyclotransferase; PFS, progression-free survival. 

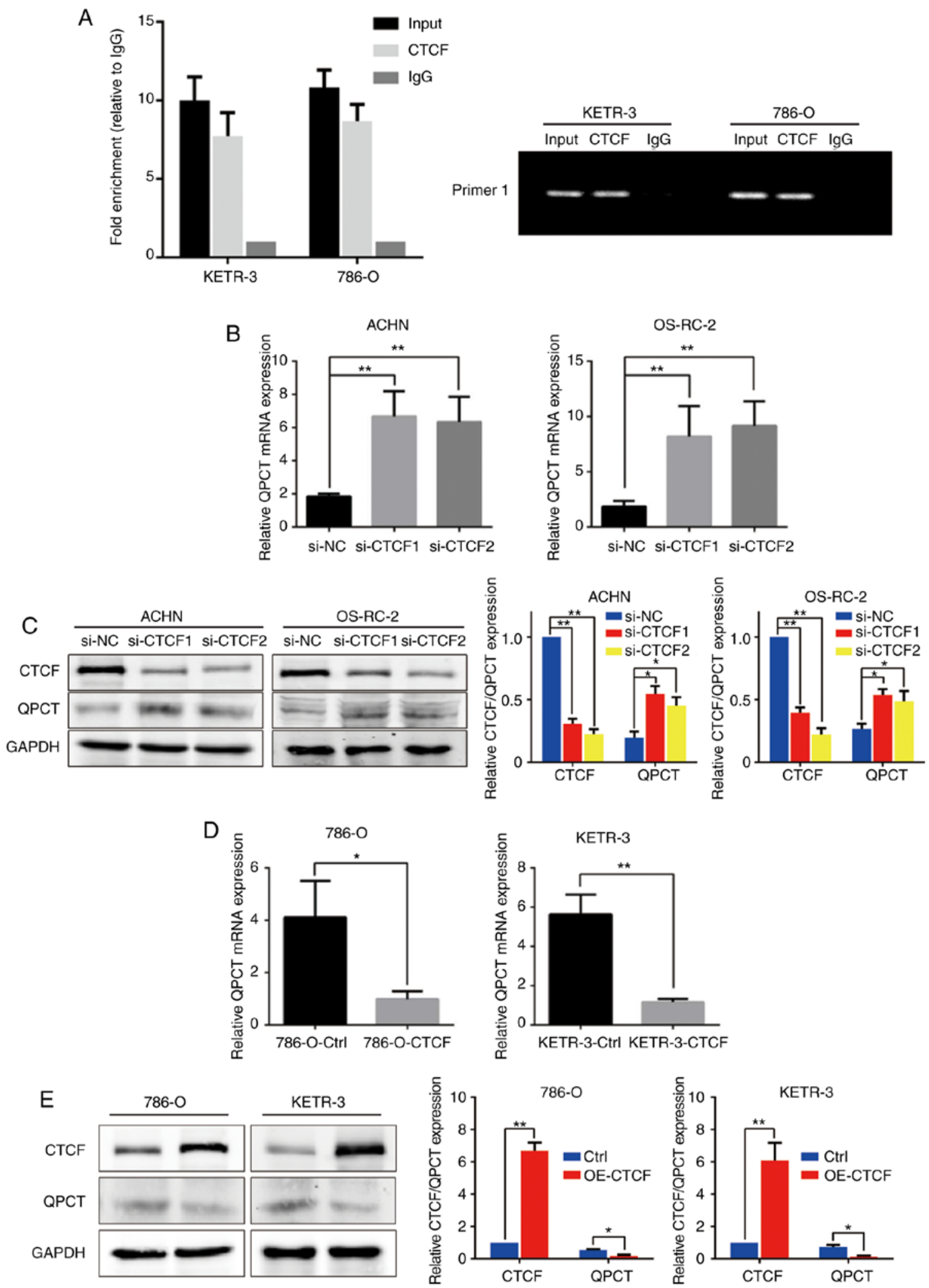

Figure 2. CTCF binds to the QPCT promoter region, negatively regulating its expression. (A) ChIP analysis demonstrated that CTCF binds to the promoter region of QPCT. (B) QPCT mRNA expression in ACHN and OS-RC-2 cells $72 \mathrm{~h}$ following CTCF knockdown, and in a control group ( $\mathrm{n}=3$ ). (C) QPCT protein expression in ACHN and OS-RC-2 cells $72 \mathrm{~h}$ following CTCF knockdown, and in a control group ( $\mathrm{n}=3$ ). (D) QPCT mRNA expression in CTCF overexpressed 786-O and KETR-3 cells and control cells $(\mathrm{n}=3$ ). (E) QPCT protein expression in CTCF-overexpressing 786-O and KETR-3 cells and control cells ( $\mathrm{n}=3$ ). Results are presented as the mean $\pm \mathrm{SD} .{ }^{*} \mathrm{P}<0.05,{ }^{* * *} \mathrm{P}<0.01$. CTCF, CCCTC-binding factor; QPCT, glutaminyl peptide cyclotransferase.

more significant improvement in PFS after receiving sunitinib compared to the control group (Fig. 1E, right panel). However, patients with a high QPCT expression did not respond well to sunitinib treatment (Fig. 1E, middle panel). Therefore, QPCT expression was suggested as an independent predictor of the sunitinib response in patients with RCC. 
A

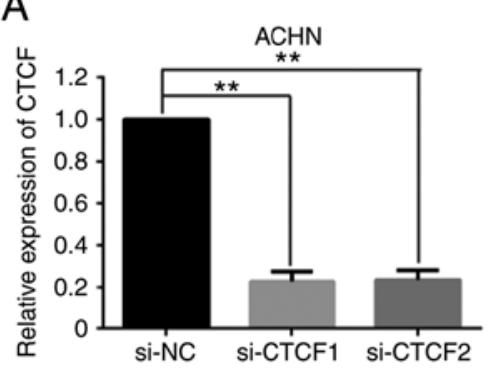

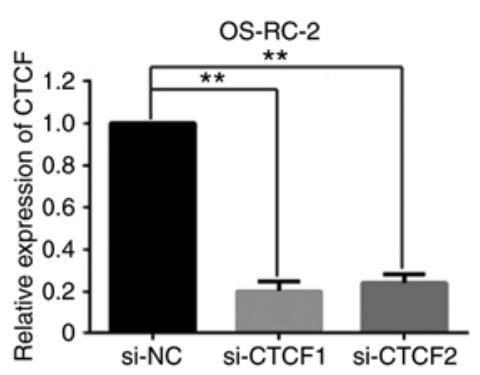

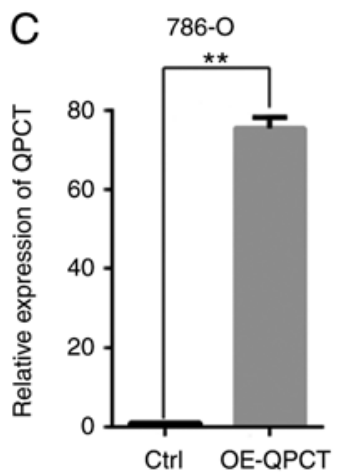

E
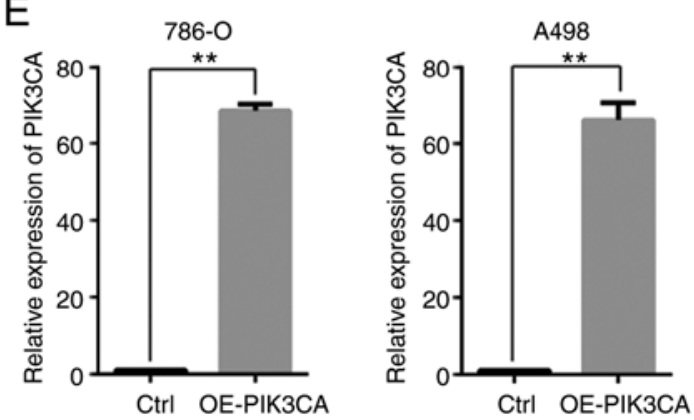

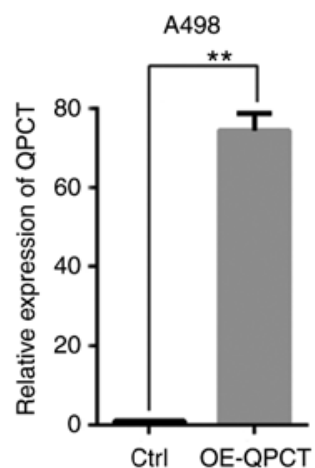

B
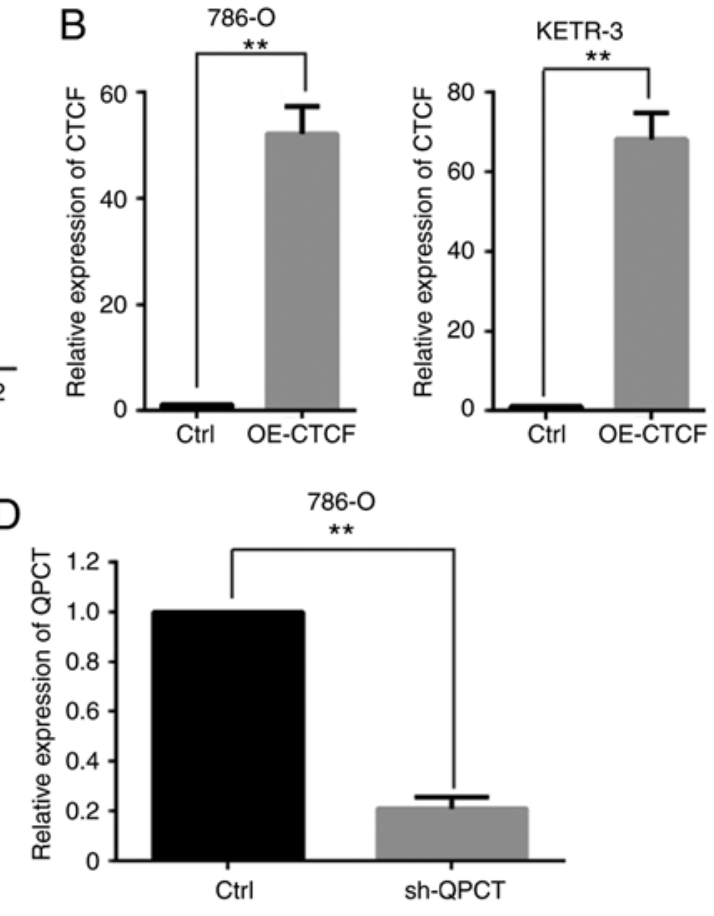
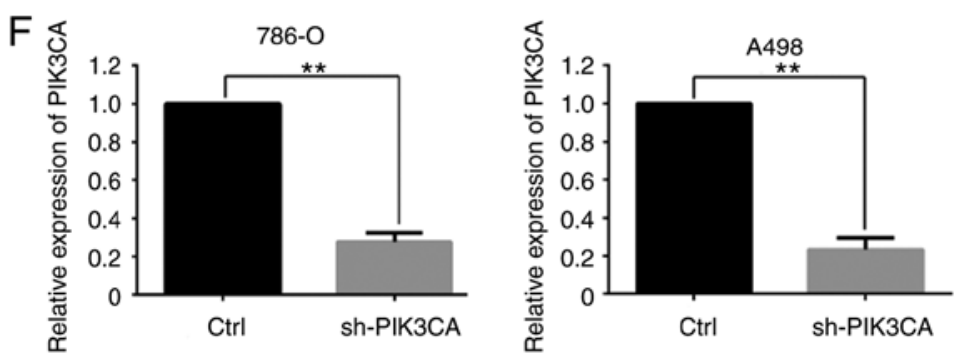

Figure 3. (A) RT-qPCR of CTCF siRNA interference efficiency in ACHN and OS-RC-2 cells. (B) RT-qPCR of lentivirus-CTCF overexpression efficiency in 786-O and KETR-3 cells. (C) RT-qPCR of lentivirus-QPCT overexpression efficiency in 786-O and A498 cells. (D) RT-qPCR of QPCT shRNA interference efficiency in 786-O cells. (E) RT-qPCR of lentivirus-PIK3CA overexpression efficiency in 786-O and A498 cells. (F) RT-qPCR of PIK3CA shRNA interference efficiency in 786-O and A498 cells. CTCF, CCCTC-binding factor; QPCT, glutaminyl peptide cyclotransferase. Results are presented as the mean \pm SD. ${ }^{* *} \mathrm{P}<0.01$.

In a previous study, the authors found that the downregulation of QPCT expression enhanced the sensitivity if RCC to sunitinib, while its overexpression promoted RCC resistance to sunitinib in vitro and in vivo (14). However, the mechanisms through which QPCT induces sunitinib resistance in RCC remain unclear. Thus, the present study aimed to elucidate these mechanisms.

CTCF binds to the QPCT promoter region, negatively regulating its expression. Through transcription factor prediction, it was found that CTCF may be one of the transcription factors regulating QPCT expression. Through ChIP assay, it was confirmed that CTCF could bind to the QPCT promoter region; the possible binding site was $-1,050 \mathrm{bp}$ of the ATG transcription start codon (Fig. 2A). To thoroughly explore the function of CTCF, the expression of CTCF was examined in RCC cell lines by RT-qPCR (Fig. SIA) and CTCF expression was then suppressed utilizing two siRNAs against CTCF in the ACHN and OS-RC-2 cell lines (Fig. 3A). QPCT expression was upregulated following interference with $\mathrm{CTCF}$ (Fig. 2B and C). Subsequently, CTCF was overexpressed in the 786-O and KETR-3 cell lines (Fig. 3B). QPCT expression was downregulated when CTCF was overexpressed (Fig. 2D and E). This indicated that CTCF negatively regulated the expression of QPCT.

Overexpression of QPCT promotes tumor angiogenesis. In the xenograft tumors formed from QPCT-overexpressing and control 786-O cells (14), it was found that the expression of CD31 and CD34 significantly increased when QPCT was overexpressed (Fig. 4A). Therefore, it was suggested that QPCT can promote angiogenesis in RCC. Moreover, one of the sunitinib targets in the treatment of RCC is the inhibition of tumor angiogenesis. Hence, it was hypothesized that when QPCT was overexpressed, the ability of sunitinib to inhibit angiogenesis would be suppressed. In order to verify this hypothesis, tube formation assays were carried out using HUVECs (the total tube length was calculated using ImageJ 
A

QPCT+sunitinib

Ctrl+sunitinib

Ctrl+vehicle

QPCT+vehicle

B
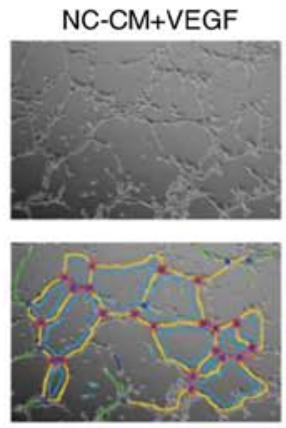

NC-CM+VEGF
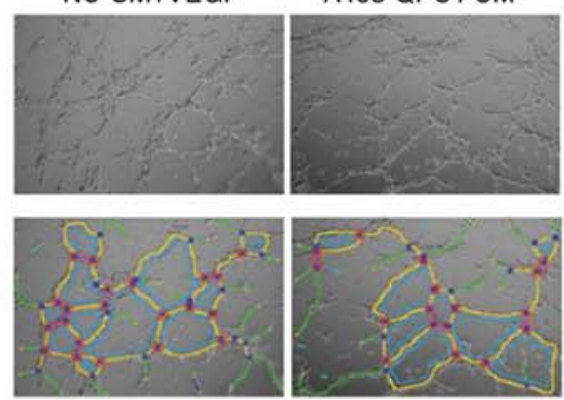

C
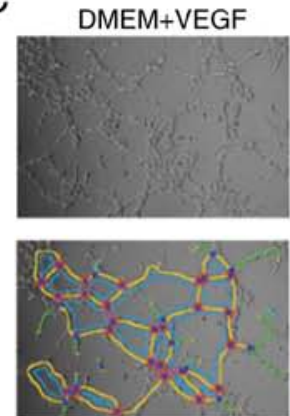

786-O-QPCT-CM
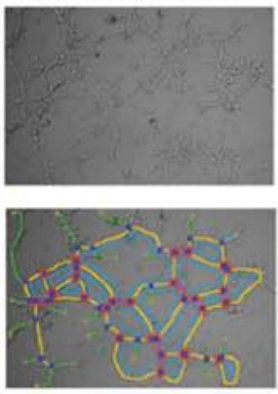

A498-QPCT-CM

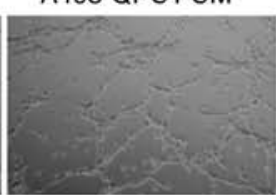

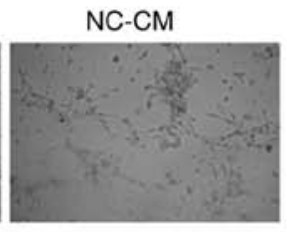
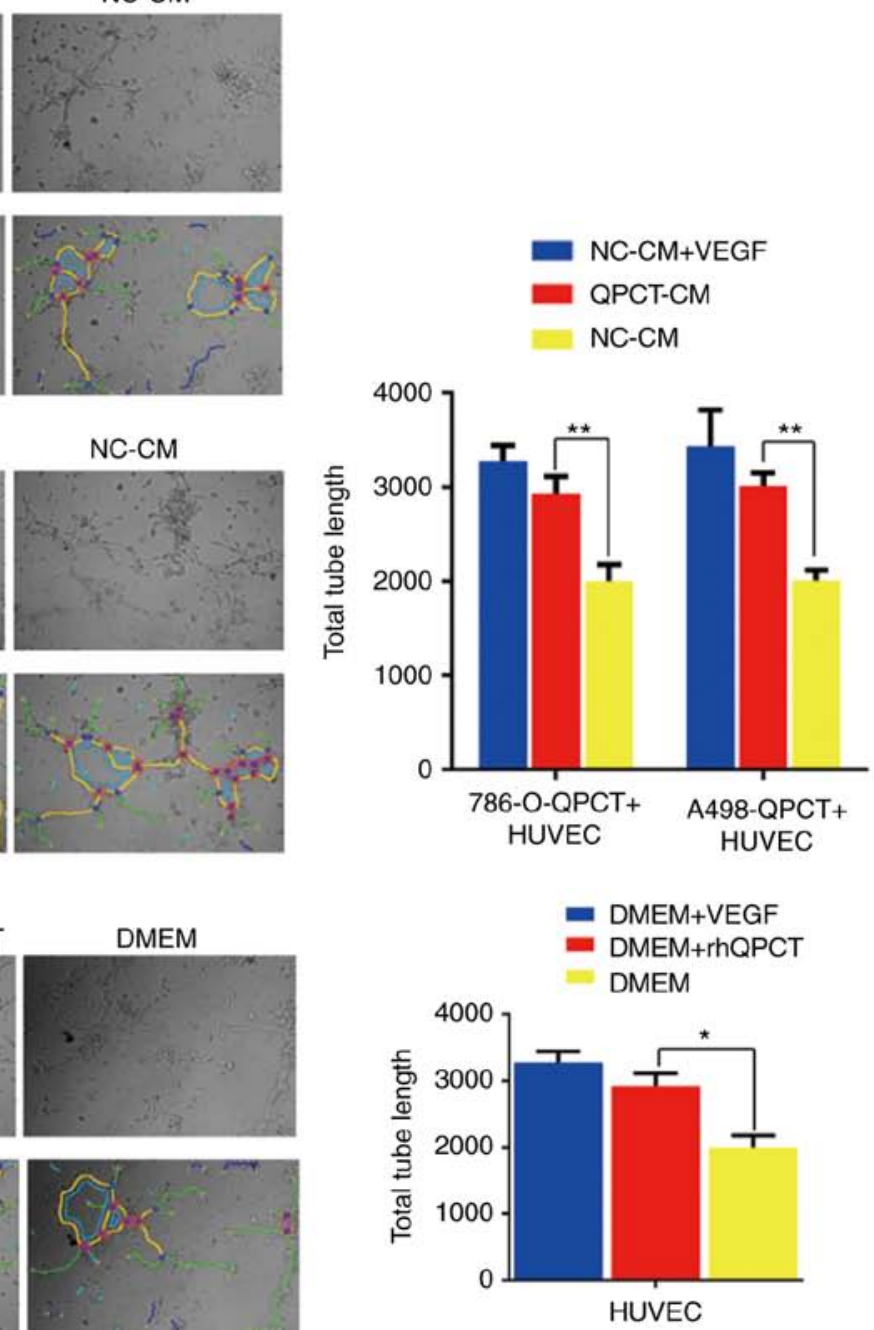

Figure 4. Overexpression of QPCT promotes tumor angiogenesis. (A) Representative immunohistochemical results of QPCT, CD31 and CD34 in xenografts. Scale bar, $100 \mu \mathrm{m}$. (B) Representative results of HUVEC tube formation cultured with the supernatant of RCC cells overexpressing QPCT. (C) Representative results of HUVEC tube formation cultured with purified QPCT cytokines (rhQPCT). The group incubated with exogenous VEGF was used as the positive control group. Total tube length was calculated using ImageJ software. Results are presented as the mean $\pm \mathrm{SD}$. ${ }^{*} \mathrm{P}<0.05,{ }^{* *} \mathrm{P}<0.01$. QPCT, glutaminyl peptide cyclotransferase; $\mathrm{CM}$, conditioned medium. 
A

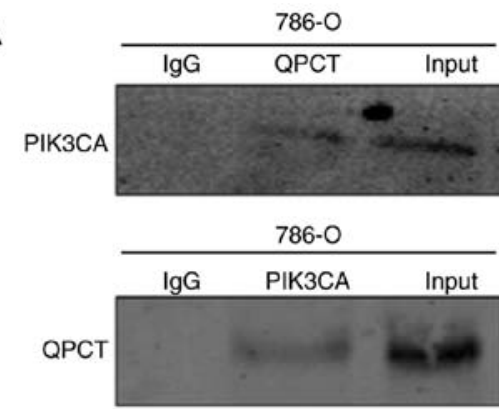

B
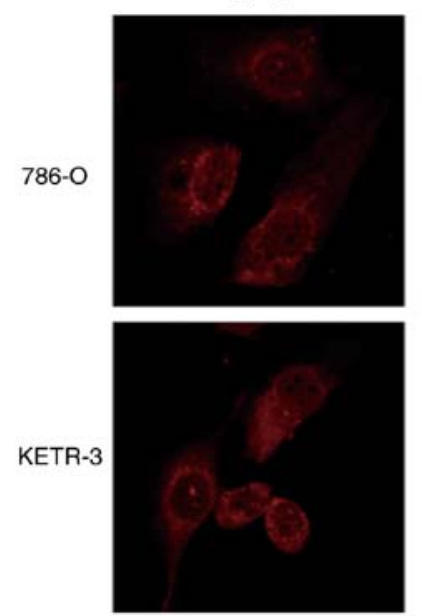

PIK3CA
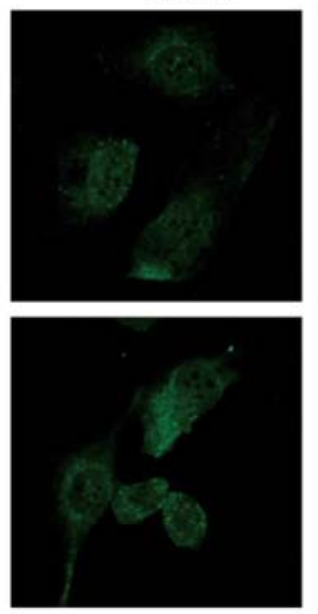

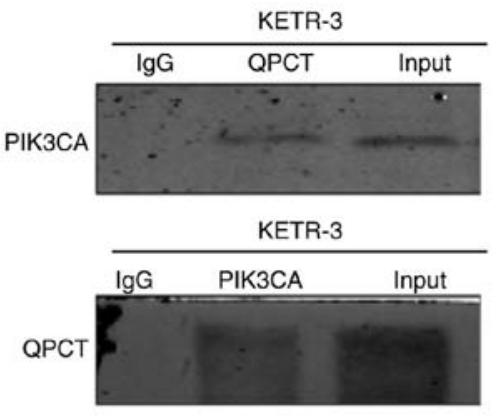

Dapi
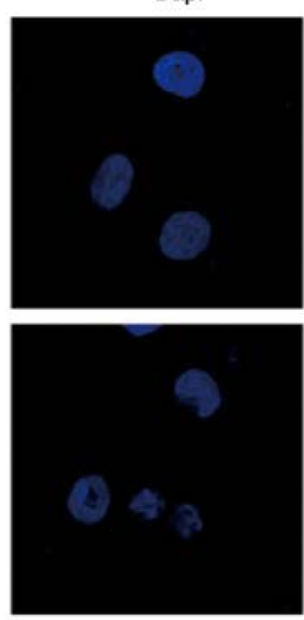
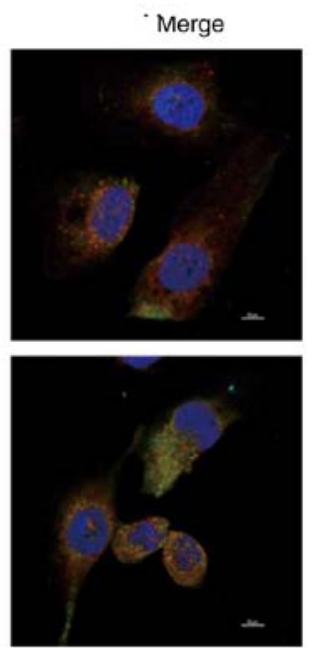

Figure 5. QPCT can bind to PIK3CA. (A) Co-immunoprecipitation of QPCT and PIK3CA in 786-O and KETR-3 cells. (B) Immunofluorescence analysis of QPCT (red) and PIK3CA (green) in OS-RC-2 and 786-O cells. Scale bar, $25 \mu \mathrm{m}$. QPCT, glutaminyl peptide cyclotransferase; PIK3CA, phosphatidylinositol4,5-bisphosphate 3-kinase catalytic subunit alpha.

software). Knowing that QPCT can be secreted extracellularly by RCC cells $(14,15)$, HUVECs were incubated with culture supernatant from RCC cells stably overexpressing QPCT (Fig. 3C). The results revealed that the HUVECs formed more tubes compared with the negative control group, while there was no significant difference between the experimental group and the positive control group (cell cultured with VEGF added to the culture medium) (Fig. 4B).

As RCC cells can secrete a variety of factors extracellularly $(11,13,14)$, the present study chose to add the purified QPCT cytokines (rhQPCT) into the HUVEC culture medium. Similarly, it was found that HUVECs cultured with rhQPCT formed more tubes compared with the negative control group (Fig. 4C).

QPCT can bind with PIK3CA. To clarify the mechanisms underlying the role of QPCT in sunitinib resistance in RCC, a human proteome microarray consisting of 20,240 full-length human proteins and $\mathrm{N}$-terminal glutathione S-transferase (GST) tags was used to search for proteins that interact with QPCT. A total of 366 proteins were detected (14). Information on proteins that may bind to QPCT is presented in Table SIV. In the Kyoto Encyclopedia of Genes and Genomes/Genome Ontology (KEGG/GO) database, it was found that QPCT bound to PIK3CA, a key proto-oncogene in the PI3K/AKT signaling pathway. The protein encoded by PIK3CA was a subunit of the PI3K enzyme. PIK3CA was involved in the PI3K/AKT pathway, which plays a crucial biological role in cell growth, proliferation, apoptosis, angiogenesis, autophagy and other cell processes. The disruption of this pathway leads to a range of diseases, including cancer (19-21). The present study verified the results of ChIP with co-IP, and PIK3CA was proven capable of combining with QPCT (Fig. 5A). QPCT co-localized with PIK3CA in the cytoplasm, as shown by immunofluorescence staining and laser confocal microscopy, thus further confirming the binding of QPCT with PIK3CA (Fig. 5B).

Overexpression of PIK3CA promotes sunitinib resistance in $R C C$. To verify the role of PIK3CA in resistance to sunitinib in RCC, the expression of PIK3CA was examined in RCC cell lines by RT-qPCR (Fig. SIB) and PIK3CA was then overexpressed in the 786-O and A498 cell lines (Fig. 3E). Subsequently, 7x10 lv-PIK3CA and lv-NC 786-O cells were inoculated subcutaneously into the left and right side of male athymic BALB/c nude mice. When the xenografts grew to $100 \mathrm{~mm}^{3}$, sunitinib (40 mg/kg/day) or saline (control) were used for intragastric administration in the mice. The results revealed that the tumor xenografts formed from RCC cells overexpressing PIK3CA exhibited worse responses to sunitinib (Fig. 6A). It was also found that the expression levels of CD31 and CD34 were upregulated in the tumor tissue overexpressing PIK3CA (Fig. 6B).

PIK3CA plays a role in angiogenesis in RCC. Similarly, data were obtained in vitro. PIK3CA expression was suppressed utilizing a shRNA against PIK3CA in the 786-O and A498 cell lines (Fig. 3F). Significantly lower tube formation was 
A
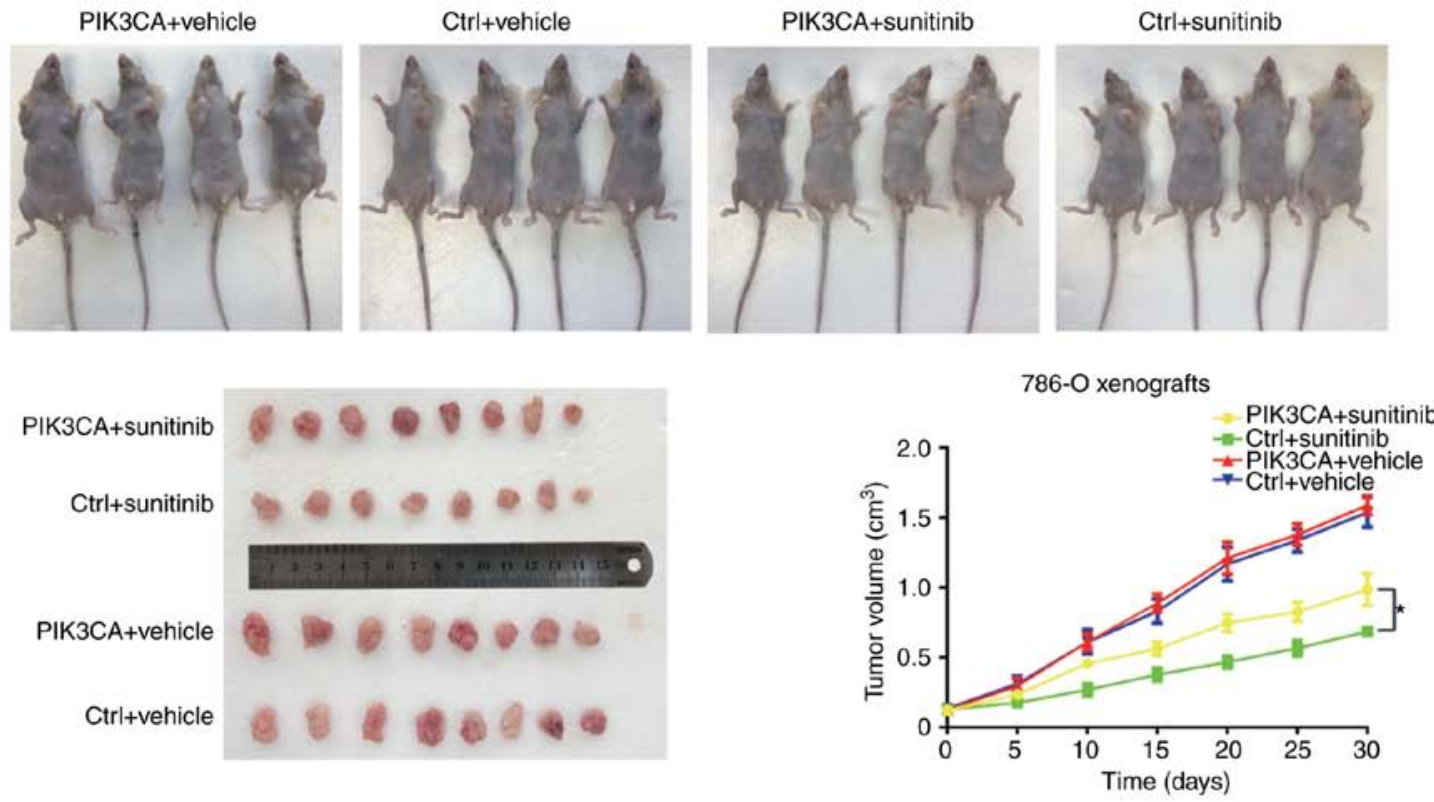

B

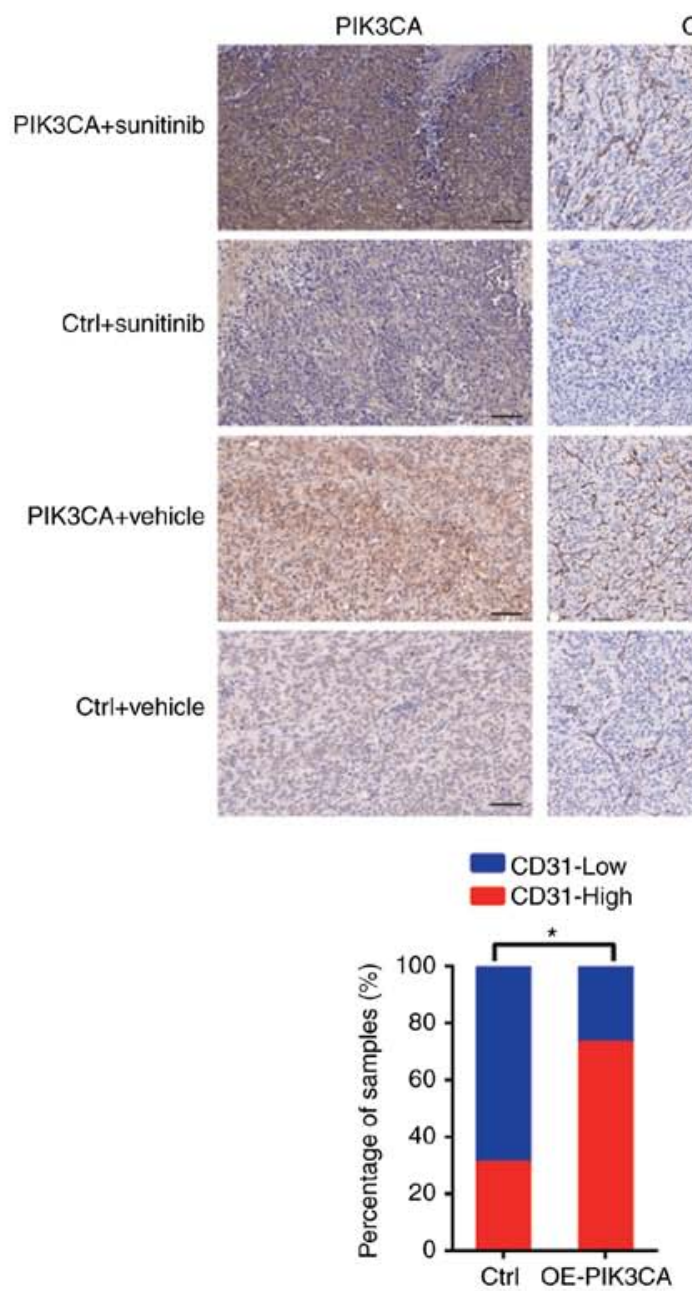

CD31
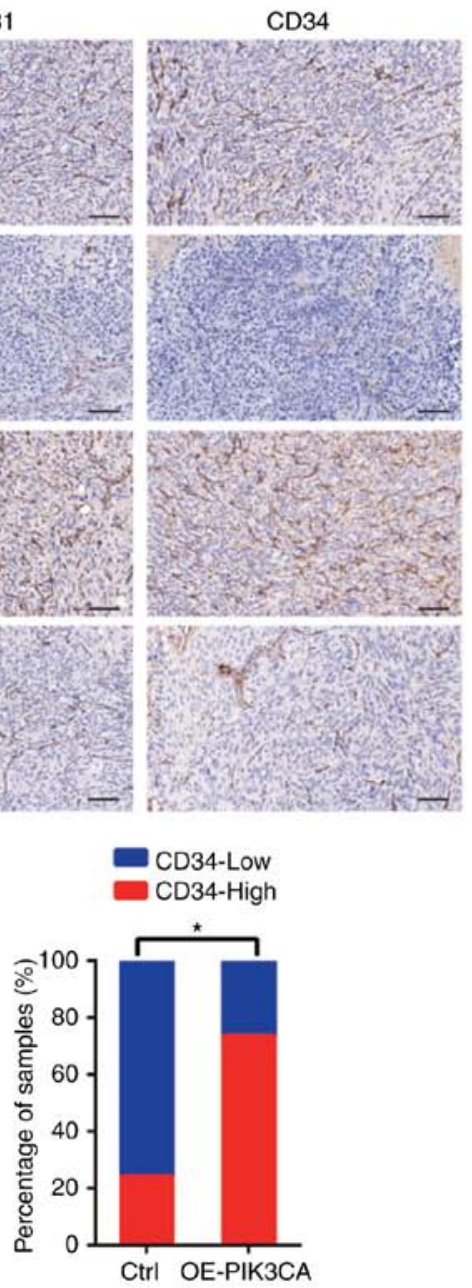

Figure 6. Overexpression of PIK3CA promotes sunitinib resistance in RCC. (A) Subcutaneous xenograft growth in nude mice under different treatment conditions (upper panel). Anatomical picture of subcutaneous xenografts in nude mice (lower left panel) and growth curve of subcutaneous xenografts (lower right panel). (B) Representative immunohistochemical results of PIK3CA, CD31 and CD34 in xenografts. Scale bar, $100 \mu \mathrm{m}$. Results are presented as the mean \pm SD. ${ }^{*} \mathrm{P}<0.05$. PIK3CA, phosphatidylinositol-4,5-bisphosphate 3-kinase catalytic subunit alpha; RCC, renal cell carcinoma.

found when the HUVECs were incubated with the culture supernatant of RCC cells in which PIK3CA was knocked down compared with the positive group (Fig. 7A). By contrast, tube formation increased when the HUVECs were incubated with the culture supernatant of RCC cells overexpressing PIK3CA compared with the negative control group (Fig. 7B). 

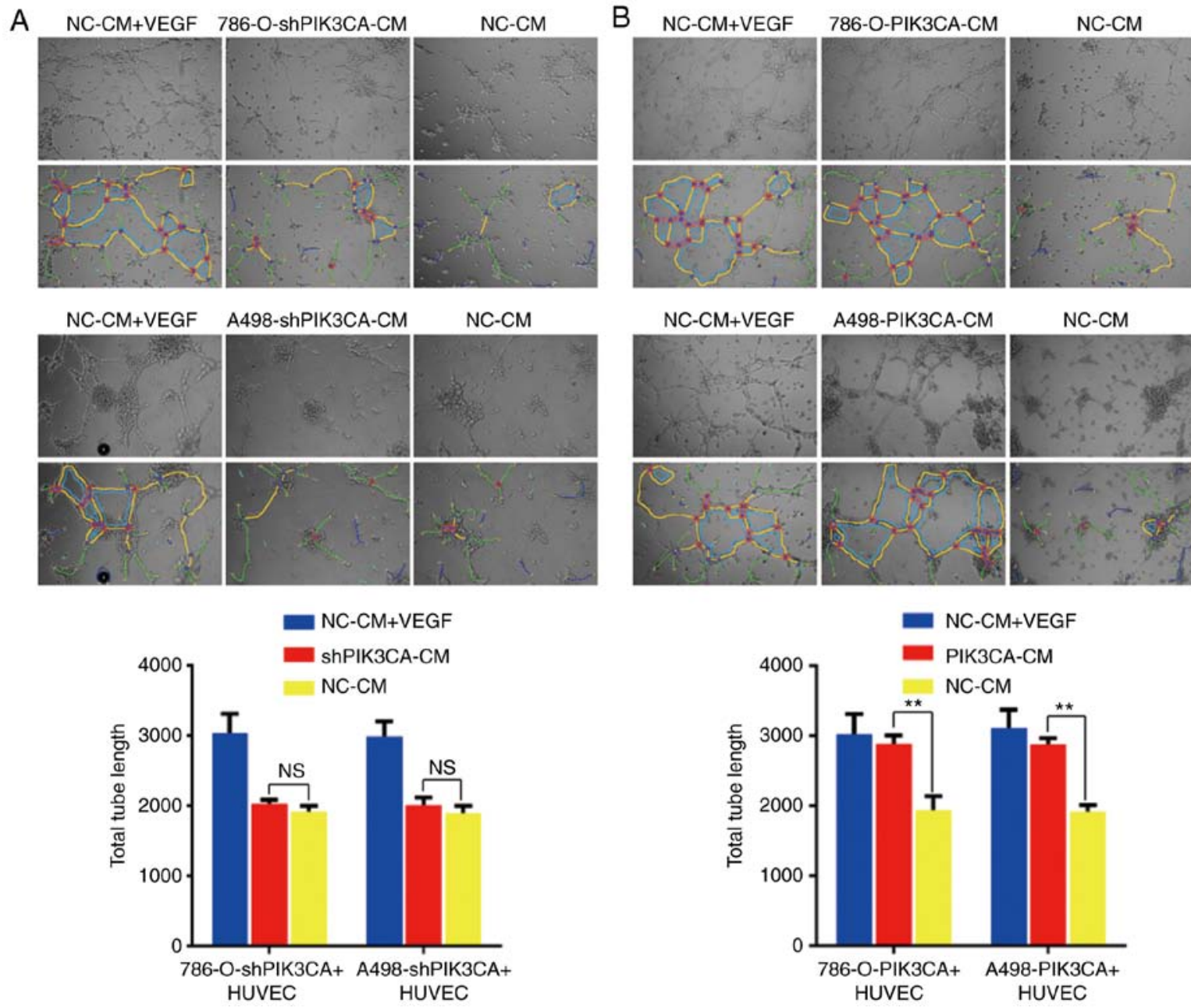

Figure 7.PIK3CA plays a role in angiogenesis in RCC.(A) Representative results of HUVEC tube formation cultured with the supernatant of PIK3CA-knockdown RCC cells. (B) Representative results of HUVEC tube formation cultured with the supernatant of PIK3CA-overexpressing RCC cells. The group with VEGF added to the culture medium was used as the positive control. Total tube length was calculated by ImageJ software. Results are presented as the means \pm SD. ${ }^{* *} \mathrm{P}<0.01$. PIK3CA, phosphatidylinositol-4,5-bisphosphate 3-kinase catalytic subunit alpha; RCC, renal cell carcinoma; $\mathrm{CM}$, conditioned medium.

These results were consistent with those of certain previous studies suggesting that PIK3CA overexpression promotes angiogenesis (19-22).

QPCT enhances the stability of PIK3CA by reducing the degradation of PIK3CA ubiquitination. As QPCT mediates the post-translational modification of proteins by converting $\mathrm{N}$-terminal glutamate to pyroglutamate, it renders proteins more resistant to protease degradation. The present study found that PIK3CA expression was upregulated in RCC cells that stably overexpressed QPCT (Fig. 8A), while PIK3CA expression was downregulated when QPCT was knocked down (Fig. 8B). In addition, immunohistochemistry of xenograft tumors derived from QPCT-overexpressing and control 786-O cells also indicated that PIK3CA expression was upregulated in response to QPCT overexpression (Fig. 8C). Through the chase experiment with CHX, it was found that QPCT inhibited the degradation of PIK3CA, and when QPCT was overexpressed, the half-life of PIK3CA was significantly prolonged, indicating that the overexpression of QPCT enhanced the stability of PIK3CA (Fig. 8D). In addition, ubiquitin analysis revealed that the overexpression of QPCT attenuated PIK3CA ubiquitin in the sunitinib-treated RCC cells (Fig. 8E).
QPCT regulates angiogenesis through PIK3CA, and $p$-AKT levels are upregulated when PIK3CA is overexpressed. By the 'rescue method', it was found that the knockdown of PIK3CA in QPCT-overexpressing cells weakened the tube formation ability of HUVECs (Fig. 9A). Furthermore, the overexpression of PIK3CA in cells in which QPCT was knocked down promoted tube formation of HUVECs (Fig. 9B). Therefore, it was suggested that QPCT promotes angiogenesis through PIK3CA. In the PIK3CA-overexpressing RCC cells, although the total AKT expression remained unaltered, p-AKT levels were upregulated, representing the activation of the PI3K/AKT signaling pathway (Fig. 9C). On the whole, it was confirmed that QPCT enhanced the stability of PIK3CA by reducing its ubiquitination, thus promoting angiogenesis and resulting in sunitinib resistance in RCC.

\section{Discussion}

The mechanisms of drug resistance can be divided into the following: The activation of angiogenic signaling pathways, the change in the tumor microenvironment, the enhancement of tumor invasion and metastasis, the role of microRNAs and the activation of other signaling pathways (23). Previous 
A

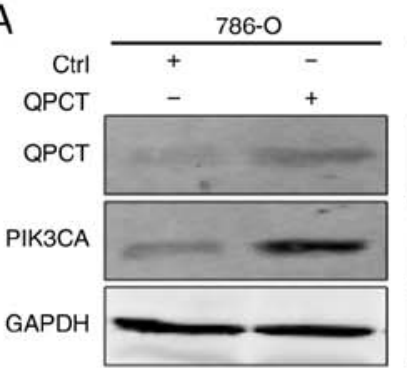

B
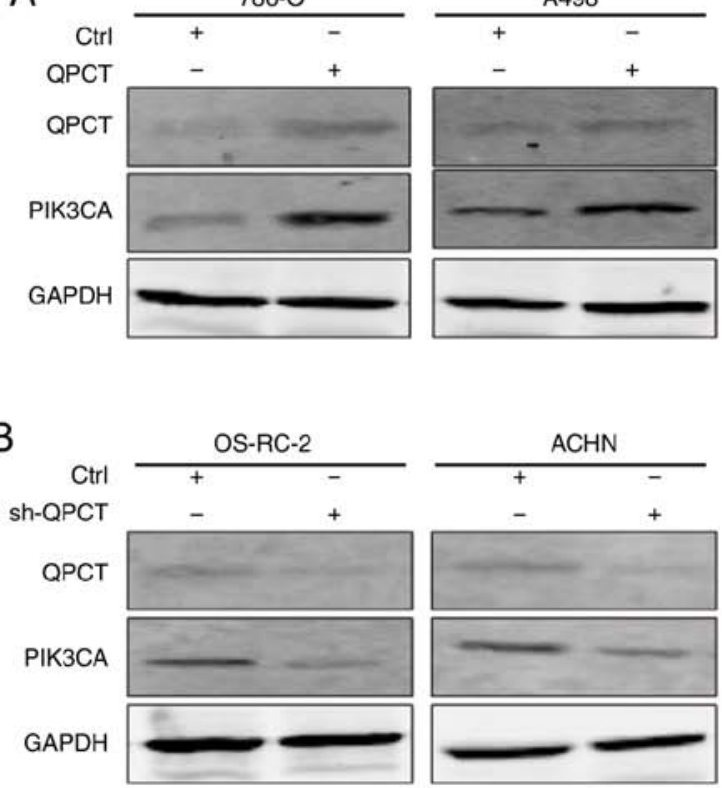
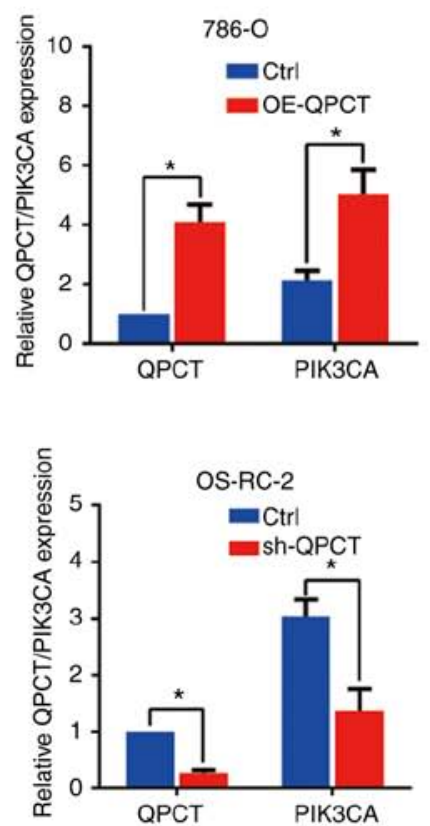

QPCT
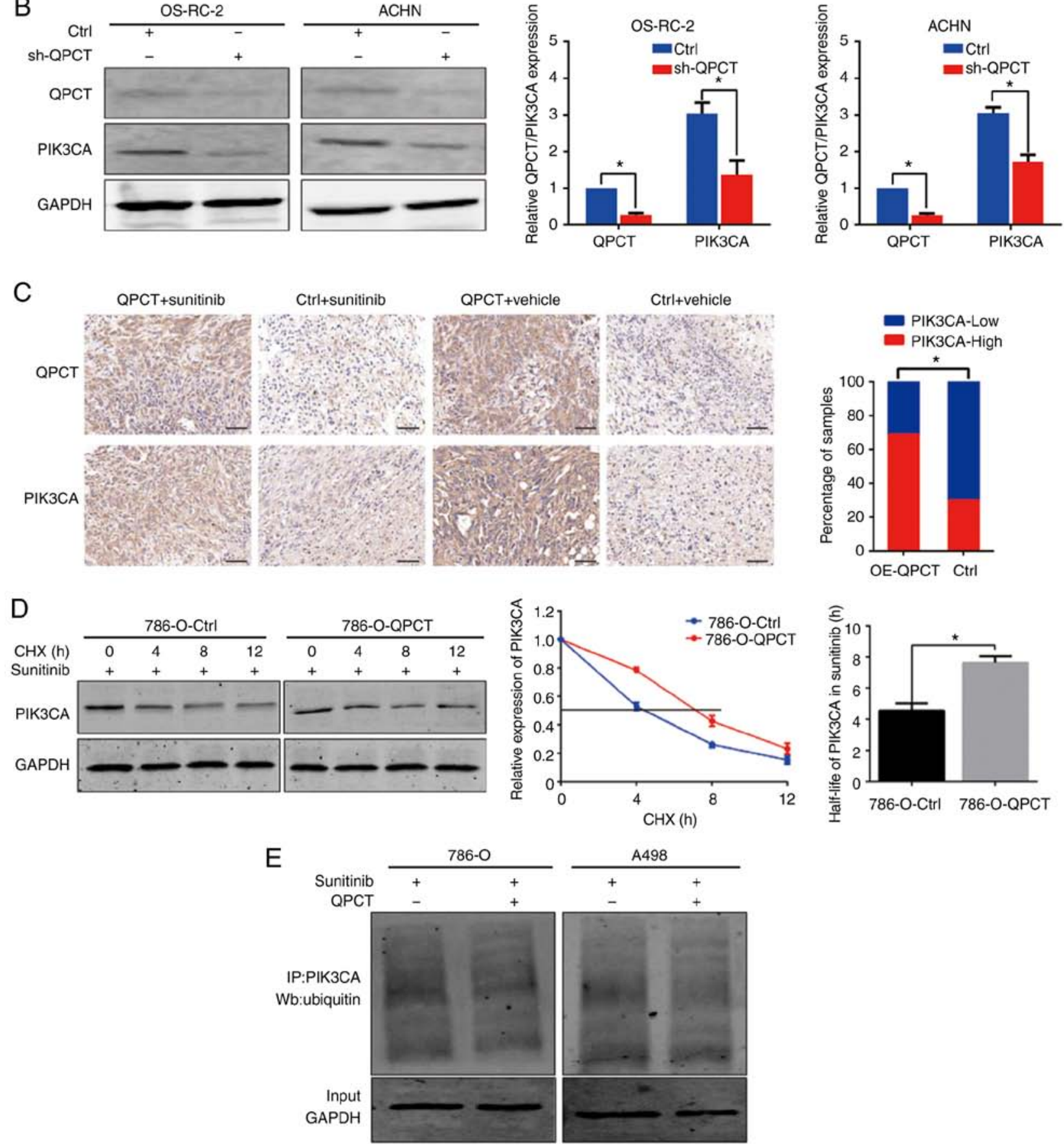

Figure 8. QPCT enhances the stability of PIK3CA by reducing the degradation of PIK3CA ubiquitination. (A) Results of western blot analysis of QPCT and PIK3CA in QPCT-overexpressing 786-O and A498 cells and control cells. (B) Results of western blot analysis of QPCT and PIK3CA in OS-RC-2 and ACHN cells transfected with sh-QPCT or sh-NC. (C) Representative immunohistochemical results of QPCT and PIK3CA in xenografts. Scale bar, $100 \mu \mathrm{m}$. (D) Western blot analysis of PIK3CA in QPCT overexpressed 786-O cells and control cells treated with CHX and sunitinib (5 $\mu \mathrm{m})$ for a different period of time. (E) Western blot analysis of PIK3CA ubiquitination in QPCT overexpressed 786-O and A498 cells and control cells after sunitinib $(5 \mu \mathrm{M})$ treatment for $48 \mathrm{~h}$. GAPDH was used as the load control. Results are presented as the mean \pm SD. ${ }^{*} \mathrm{P}<0.05$. QPCT, glutaminyl peptide cyclotransferase; PIK3CA, phosphatidylinositol-4,5-bisphosphate 3-kinase catalytic subunit alpha; CHX, cycloheximide. 
A

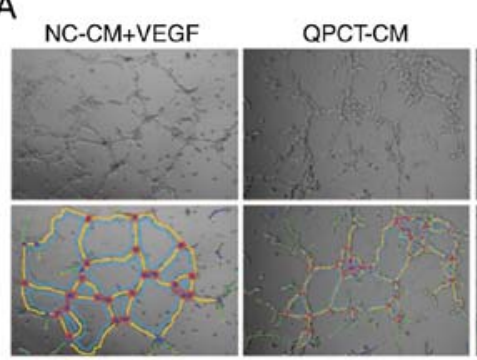

B

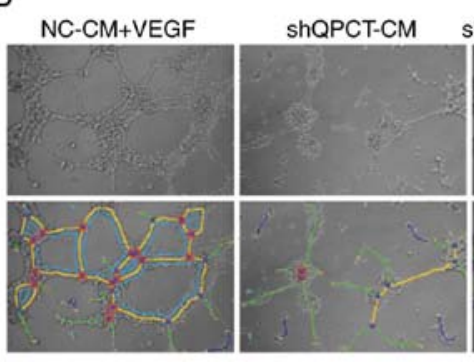

C

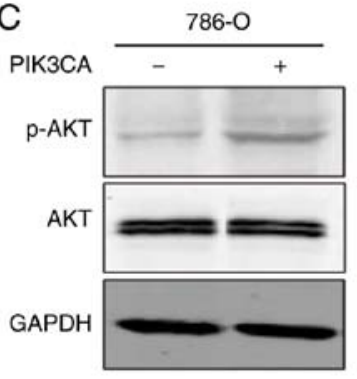

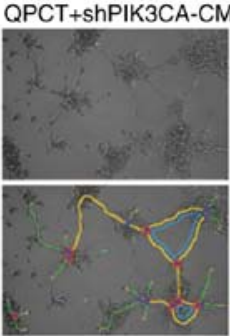

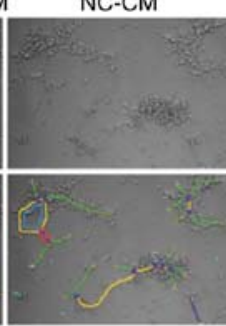

ShQPCT+ShPIK3CA-CM

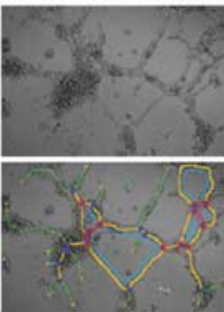

A498

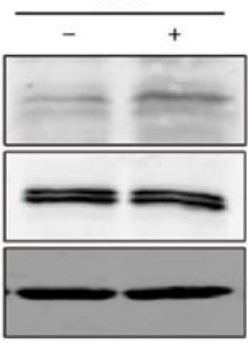

NC-CM
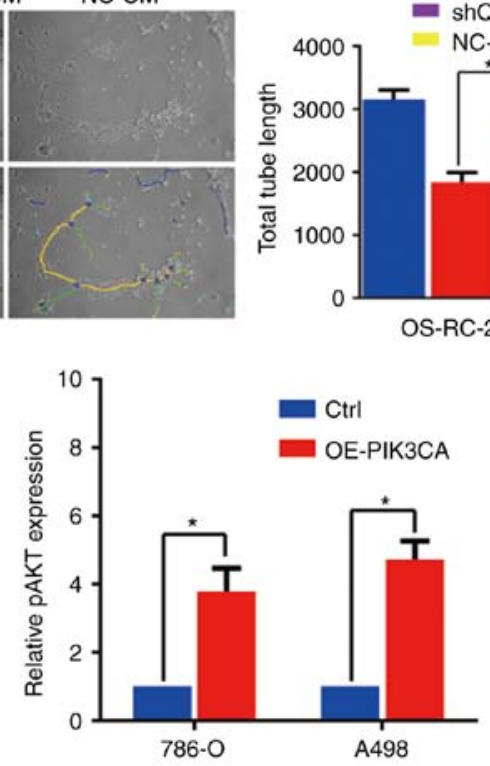

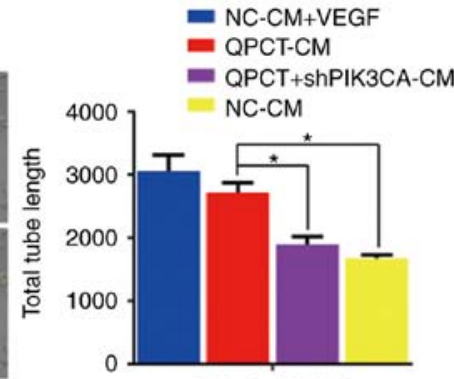

768-O+HUVEC

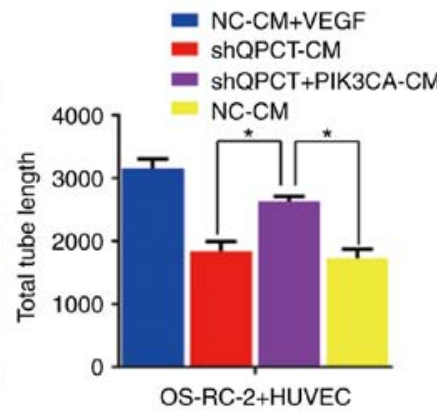

Figure 9. QPCT regulates angiogenesis through PIK3CA. (A) Representative results of HUVEC tube formation cultured with the supernatant of QPCT-overexpressing+PIK3CA-knockdown RCC cells.(B) Representative results of HUVEC tube formation cultured with the supernatant of QPCT-knockdown +PIK3CA-overexpressing RCC cells. (C) Representative results of western blot analysis of AKT and p-AKT in PIK3CA overexpressed 786-O and A498 cells and control cells. The group with VEGF added into the culture medium was used as the positive control. Total tube length was calculated using ImageJ software. Results are presented as the means $\pm \mathrm{SD}$. ${ }^{*} \mathrm{P}<0.05$. QPCT, glutaminyl peptide cyclotransferase; PIK3CA, phosphatidylinositol-4,5-bisphosphate 3-kinase catalytic subunit alpha; CM, conditioned medium.

research has demonstrated that angiogenic factors are upregulated in patients resistant to sunitinib (24). In fact, anti-angiogenesis-induced hypoxia activates the mTOR signaling pathway, promotes HIF production and activates the transcription of HRE-containing genes, including VEGF, PDGF, transforming growth factor (TGF)- $\alpha$, erythropoietin (EPO), matrix metalloproteinase (MMP)-1, epidermal growth factor receptor (EGFR), hepatocyte growth factor receptor (HGFR)/cMET, cyclin D1, stromal cell-derived factor (SDF)1 and CXCR4. In addition, the key role of the changes in the tumor microenvironment in sunitinib-resistance RCC has also been confirmed (25). Some researchers have highlighted the role of pericytes in sunitinib resistance in RCC. Pericytes grow and cover endothelial cells after inhibiting VEGF (26).

The QPCT gene encodes glutamylpeptidyl transferase, which modifies proteins by converting N-terminal glutamate to pyroglutamine. This renders the protein more resistant to protease degradation, making it hydrophobic, neurotoxic, and easier to aggregate (15). At present, there are limited studies available on QPCT in tumors. Few have reported the role of QPCT in thyroid cancer (27-29) and melanoma (30). In the present study, it was found that QPCT was strongly associated with sunitinib resistance in RCC. QPCT expression was increased in sunitinib-resistant RCC tissues and plasma, and high QPCT levels predicted a poor response to sunitinib in patients with RCC. It was further confirmed that the downregulation of QPCT enhanced the sensitivity of RCC cells to sunitinib, while its overexpression promoted resistance in vitro and in vivo (14). Through transcription factor prediction and ChIP assay verification, it was found that the transcription factor CTCF binds to the QPCT promoter region and negatively regulated its expression. CTCF, Zinc-finger protein, is a multifunctional transcription factor widely expressed in eukaryotes (31). CTCF is a nuclear protein, which is widely spread across cell types. CTCF is a multifunctional transcription factor that regulates gene expression through various mechanisms, including the recruitment of other coactivators and binding to target gene promoter regions. Genetic alterations in CTCF have been found in a number of types of cancer, such as liver cancer, lung cancer, stomach cancer and breast cancer (32-34). The elimination of CTCF confirms the multifunctional state of the protein, which is an important factor in transcriptional regulation, unique ring formation, and maintenance of chromatin structure, and is involved in protein complexes, such as adhesion proteins in 
interchromatin and chromatin inner rings (32-35). Abnormal CTCF expression has been found to induce a number of diseases or disorders, including various types of cancer $(31,36)$. In particular, the downregulation of CTCF is positively associated with the dysregulation of $\mathrm{CpG}$ methylation patterns around genes known to be involved in tumorigenesis such as tumor protein P53 (TRp53), DNA methyltransferase 1a (DNMT4a), Runt-related transcription factor 1 (RUNX1) and CTCF] (37). DNA methylation in $\mathrm{CpG}$ regions near CTCF regulatory genes (including oncogenes) leads to unusable CTCF binding (31). This is consistent with our previous research results (14). It was found that in sunitinib-resistant RCC tissues, the methylation level of the QPCT promoter region was significantly changed (14), which may affect the binding of CTCF with the QPCT promoter region, thus affecting the expression of QPCT. Cancer genome sequencing revealed multiple acquired mutations in CTCF, which turned out to be a tumor suppressor gene. Thus, tumor growth is enhanced in the absence of CTCF regulation of the relevant genes. The dysfunction of CTCF can alter many cancer-related genes epigenetically $(31,38)$.

Through human proteome microarray, co-IP, immunofluorescence staining and confocal laser microscopy observation, the present study found that QPCT bound to PIK3CA, and $\mathrm{PI} 3 \mathrm{~K} / \mathrm{Akt} / \mathrm{mTOR}$ was one of the main intracellular signaling pathways. PI3K signals regulate various cellular functions, including translational regulation of cell proliferation, survival, protein synthesis, glucose metabolism, cell migration and angiogenesis (39-43). The disruption of this pathway leads to a range of diseases, including cancer (44). PI3K/Akt/mTOR pathway plays an important role in the regulation of angiogenesis in normal and cancerous tissues (19-22,39). PI3K proteins are a family of lipid kinases that are activated in growth factor receptor tyrosine kinases (RTKs) and G-protein coupled receptor signaling. PIK3CA is the most commonly associated gene in huma cancers that has been shown to contain oncogenic mutations or amplifications (45).

In conclusion, the data of the present study suggested that QPCT, which was negatively regulated by CTCF, could enhance the stability of PIK3CA by reducing its ubiquitination, thus promoting angiogenesis and leading to sunitinib resistance in RCC. Therefore, QPCT and PIK3CA may prove to be novel targets for the treatment or reversal of sunitinib resistance in RCC.

\section{Acknowledgements}

Not applicable.

\section{Funding}

The present study was supported by the National Natural Science Foundation of China (grant nos. 82002700 and 82072836), Postdoctoral Science Foundation of China (grant nos. 2020M673678), and Postdoctoral Science Foundation of Jiangsu Province (grant no. 2020Z363).

\section{Availability of data and materials}

The datasets used and/or analyzed during the current study are available from the corresponding author on reasonable request.

\section{Authors' contributions}

$\mathrm{TZ}$ and $\mathrm{YZ}$ conducted all experiments and analyzed the data. $\mathrm{XY}, \mathrm{HH}$ and QW provided support with the experimental techniques. TZ, QW, SG, HH, SX, BD, JD and JG collected the clinical data. TZ, HH and SX wrote the manuscript. TZ, YZ and $\mathrm{HH}$ confirmed the authenticity of all the raw data. WZ, LW and LQ provided the clinical samples, contributed to manuscript revision, conceived the study and supervised all the experiments. All authors have read approved the final manuscript.

\section{Ethics approval and consent to participate}

All patients signed the informed consent before participating in the study, and the plan was approved by the Ethics Committee of Jinling Hospital. The accession number for this approval was 2020DZGZRZX-008. The animal experiments were performed in accordance with relevant guidelines and regulations for the care and use of laboratory animals, with the approval of the Institutional Animal Care and Use Committee at Jinling Hospital. The accession number for this approval was 2020JLHGKJDWLS-47.

\section{Patient consent for publication}

Not applicable.

\section{Competing interests}

The authors declare that they have no competing interests.

\section{References}

1. Capitanio U and Montorsi F: Renal cancer. Lancet 387: 894-906, 2016.

2. Siegel RL, Miller KD and Jemal A: Cancer statistics, 2010. CA Cancer J Clin 60: 277-300, 2010.

3. Ljungberg B, Campbell SC, Choi HY, Jacqmin D, Lee JE, Weikert S and Kiemeney LA: The epidemiology of renal cell carcinoma. Eur Urol 60: 615-621, 2011.

4. Ljungberg B, Bensalah K, Canfield S, Dabestani S, Hofmann F, Hora M, Kuczyk MA, Lam T, Marconi L, Merseburger AS, et al: EAU guidelines on renal cell carcinoma: 2014 update. Eur Urol 67: 913-924, 2015.

5. Gupta K, Miller JD, Li JZ, Russell MW and Charbonneau C: Epidemiologic and socioeconomic burden of metastatic renal cell carcinoma (mRCC): A literature review. Cancer Treat Rev 34: 193-205, 2008.

6. Motzer RJ, Bacik J, Murphy BA, Russo P and Mazumdar M: Interferon-alfa as a comparative treatment for clinical trials of new therapies against advanced renal cell carcinoma. J Clin Oncol 20: 289-296, 2002.

7. Motzer RJ, Hutson TE, Tomczak P, Michaelson MD, Bukowski RM, Rixe O, Oudard S, Negrier S, Szczylik C, Kim ST, et al: Sunitinib versus interferon alfa in metastatic renal-cell carcinoma. N Engl J Med 356: 115-124, 2007.

8. National Comprehensive Cancer Network ( NCCN): NCCNClinical Practice Guidelines in Oncology: Kidney Cancer. Version 1.2017. www.nccn.org. Accessed February 6, 2017.

9. Escudier B, Porta C, Schmidinger M, Rioux-Leclercq N, Bex A, Khoo V, Gruenvald V and Horwich A; ESMO Guidelines Committee: Renal cell carcinoma: ESMO clinical practice guidelines for diagnosis, treatment and follow-up. Ann Oncol 27 (Suppl 5): v58-v68, 2016.

10. Christensen JG: A preclinical review of sunitinib, a multitargeted receptor tyrosine kinase inhibitor with anti-angiogenic and antitumour activities. Ann Oncol 18 (Suppl 10): x3-10, 2007.

11. Faivre S, Demetri G, Sargent W and Raymond E: Molecular basis for sunitinib efficacy and future clinical development. Nat Rev Durg Discov 6: 734-745, 2007. 
12. Oudard S and Elaidi RT: Sequential therapy with targeted agents in patients with advanced renal cell carcinoma: Optimizing patient benefit. Cancer Treat Rev 38: 981-987, 2012.

13. Molina AM, Lin X, Korytowsky B, Matczak E, Lechuga MJ, Wiltshire R and Motzer RJ: Sunitinib objective response in metastatic renal cell carcinoma: Analysis of 1059 patients treated on clinical trials. Eur J Cancer 50: 351-358, 2014.

14. Zhao T, Bao Y, Gan X, Wang J, Chen Q, Dai Z, Liu B, Wang A, Sun S, Yang F and Wang L: DNA methylation-regulated QPCT promotes sunitinib resistance by increasing HRAS stability in renal cell carcinoma. Theranostics 9: 6175-6190, 2019.

15. Kehlen A, Haegele M, Menge K, Gans K, Immel UD, Hoang-Vu C, Klonisch T and Demuth HU: Role of glutaminy cyclases in thyroid carcinomas. Endocr Relat Cancer 20: 79-90, 2013.

16. Livak KJ and Schmittgen TD: Analysis of relative gene expression data using real-time quantitative PCR and the 2(-Delta Delta C(T)) method. Methods 25: 402-408, 2001.

17. Yu X, Petritis B and LaBaer J: Advancing translational research with next-generation protein microarrays. Proteomics 16 : $1238-1250,2016$

18. Shen SM, Ji Y, Zhang C, Dong SS, Yang S, Xiong Z, Ge MK, Yu Y, Xia L, Guo M, et al: Nuclear PTEN safeguards pre-mRNA splicing to link Golgi apparatus for its tumor suppressive role. Nat Commun 9: 2392, 2018.

19. Wu Z, Ai X, Hu H, Wang S, Wang Y, Kang F, Ouyang C and Zhu J: Hematopoietic-substrate-1 associated protein X-1 (HAX-1) regulates liver cancer cells growth, metastasis, and angiogenesis through Akt. Cancer Biol Ther 20: 1223-1233, 2019.

20. Zhang T, Yao Y, Wang J, Li Y, He P, Pasupuleti V, Hu Z, Jia X, Song Q, Tian XL, et al: Haploinsufficiency of Klippel-Trenaunay syndrome gene Aggf1 inhibits developmental and pathological angiogenesis by inactivating PI3K and AKT and disrupts vascular integrity by activating VE-cadherin. Hum Mol Genet 25: 5094-5110, 2016

21. Zhang L, Yang N, Katsaros D, Huang W, Park JW, Fracchioli S Vezzani C, Rigault de la Longrais IA, Yao W, Rubin SC and Coukos G: The oncogene phosphatidylinositol 3'-kinase catalytic subunit alpha promotes angiogenesis via vascular endothelial growth factor in ovarian carcinoma. Cancer Res 63: 4225-4231, 2003

22. Murillo MM, Zelenay S, Nye E, Castellano E, Lassailly F, Stamp G and Downward J: RAS interaction with PI3K p110o is required for tumor-induced angiogenesis. J Clin Invest 124 3601-3611, 2014

23. Joosten SC, Hamming L, Soetekouw PM, Aarts MJ, Veeck J, van Engeland $\mathrm{M}$ and Tjan-Heijnen VC: Resistance to sunitinib in renal cell carcinoma: From molecular mechanisms to predictive markers and future perspectives. Biochim Biophys Acta 1855 $1-16,2015$.

24. Fernando NT, Koch M, Rothrock C, Gollogly LK, D'Amore PA, Ryeom S and Yoon SS: Tumor Escape from endogenous, extracellular matrix-associated angiogenesis inhibitors by up-regulation of multiple proangiogenic factors. Clin Cancer Res 14: 1529-1539, 2008

25. Oudard S, Geoffrois L, Guillot A, Chevreau C, Deville JL, Falkowski S, Boyle H, Baciuchka M, Gimel P, Laguerre B, et al: Clinical activity of sunitinib rechallenge in metastatic renal cell carcinoma-Results of the REchallenge with SUnitinib in MEtastatic RCC (RESUME) Study. Eur J Cancer 62: 28-35, 2016.

26. Bergers $G$ and Hanahan D: Modes of resistance to anti-angiogenic therapy. Nat Rev Cancer 8: 592-603, 2008.

27. Jarzab B, Wiench M, Fujarewicz K, Simek K, Jarzab M, Oczko-Wojciechowska M, Wloch J, Czarniecka A, Chmielik E, Lange D, et al: Gene expression profile of papillary thyroid cancer: Sources of variability and diagnostic implications. Cancer Res 65: 1587-1597, 2005.

28. Fluge $\varnothing$, Bruland O, Akslen LA, Lillehaug JR and Varhaug JE: Gene expression in poorly differentiated papillary thyroid carcinomas. Thyroid 16: 161-175, 2006.
29. Griffith OL, Melck A, Jones SJ and Wiseman SM: Meta-analysis and meta-review of thyroid cancer gene expression profiling studies identifies important diagnostic biomarkers. J Clin Oncol 24: 5043-5051, 2006

30. Gillis JS: Microarray evidence of glutaminyl cyclase gene expression in melanoma: Implications for tumor antigen specific immunotherapy. J Transl Med 4: 27, 2006.

31. Oh S, Oh C and Yoo KH: Functional roles of CTCF in breast cancer. BMB Rep 50: 445-453, 2017.

32. Aitken SJ, Ibarra-Soria X, Kentepozidou E, Flicek P, Feig C, Marioni JC and Odom DT: CTCF maintains regulatory homeostasis of cancer pathways. Genome Biol 19: 106, 2018.

33. Bailey CG, Metierre C, Feng Y, Baidya K, Filippova GN, Loukinov DI, Lobanenkov VV, Semaan C and Rasko JE: CTCF expression is essential for somatic cell viability and protection against cancer. Int J Mol Sci 19: 3832, 2018.

34. Lee JY, Mustafa M, Kim CY and Kim MH: Depletion of CTCF in breast cancer cells selectively induces cancer cell death via p53. J Cancer 8: 2124-2131, 2017.

35. Ren G, Jin W, Cui K, Rodrigez J, Hu G, Zhang Z, Larson DR and Zhao K: CTCF-mediated enhancer-promoter interaction is a critical regulator of cell-to-cell variation of gene expression. Mol Cell 67: 1049-1058.e6, 2017

36. Höflmayer D, Steinhoff A, Hube-Magg C, Kluth M, Simon R, Burandt E, Tsourlakis MC, Minner S, Sauter G, Büscheck F, et al: Expression of CCCTC-binding factor (CTCF) is linked to poor prognosis in prostate cancer. Mol Oncol 14: 129-138, 2020.

37. Kemp CJ, Moore JM, Moser R, Bernard B, Teater M, Smith LE, Rabaia NA, Gurley KE, Guinney J, Busch SE, et al: CTCF haploinsufficiency destabilizes DNA methylation and predisposes to cancer. Cell Rep 7: 1020-1029, 2014.

38. Chan CS and Song JS: CCCTC-binding factor confines the distal action of estrogen receptor. Cancer Res 68: 9041-9049, 2008.

39. Hosseini S, Behjati F, Rahimi M, Taheri N, Khoram Khorshid H, Aghakhani Moghaddam F, Ghasemi S, Karimlou M, Sirati F and Keyhani E: Relationship between PIK3CA amplification and $\mathrm{P} 110 \alpha$ and CD34 tissue expression as angiogenesis markers in Iranian women with sporadic breast cancer. Iran J Pathol 13 447-453, 2018

40. Chou WC, Lin PH, Yeh YC, Shyr YM, Fang WL, Wang SE, Liu CY, Chang PM, Chen MH, Hung YP, et al: Genes involved in angiogenesis and mTOR pathways are frequently mutated in Asian patients with pancreatic neuroendocrine tumors. Int J Biol Sci 12: 1523-1532, 2016.

41. Riquelme I, Tapia O, Espinoza JA, Leal P, Buchegger K, Sandoval A, Bizama C, Araya JC, Peek RM and Roa JC: The Gene Expression status of the PI3K/AKT/mTOR pathway in gastric cancer tissues and cell lines. Pathol Oncol Res 22: 797-805, 2016.

42. du Rusquec P, Blonz C, Frenel JS and Campone M: Targeting the PI3K/Akt/mTOR pathway in estrogen-receptor positive HER2 negative advanced breast cancer. Ther Adv Med Oncol 12: 175883592094093,2020

43. Sasore T and Kennedy B: Deciphering combinations of $\mathrm{PI} 3 \mathrm{~K} / \mathrm{AKT} / \mathrm{mTOR}$ pathway drugs augmenting Anti-angiogenic efficacy in vivo. PLoS One 9: e105280, 2013.

44. Bader AG, Kang S and Vogt PK: Cancer-specific mutations in PIK3CA are oncogenic in vivo. Proc Natl Acad Sci USA 103: 1475-1479, 2006.

45. De Santis MC, Gulluni F, Campa CC, Martini M and Hirsch E: Targeting PI3K signaling in cancer: Challenges and advances. Biochim Biophys Acta Rev Cancer 1871: 361-366, 2019.

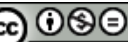

This work is licensed under a Creative Commons Attribution-NonCommercial-NoDerivatives 4.0 International (CC BY-NC-ND 4.0) License. 